\title{
Wave-induced dynamics of marine pipelines in liquefiable seabed
}

\author{
Kai Zhao ${ }^{1}$, Hao Xiong ${ }^{1}, \quad$ Guoxing Chen ${ }^{1 *}$, Dingfeng Zhao ${ }^{1}$, Weiyun Chen ${ }^{1,2}, \quad$ Xiuli Du ${ }^{3}$ \\ ${ }^{1}$ Institute of Geotechnical Engineering, Nanjing Tech University, Nanjing 210009, China \\ ${ }^{2}$ Griffith School of Engineering, Griffith University, Gold Coast Campus, Queensland, QLD 4222, Australia \\ ${ }^{3}$ Key Laboratory of Urban Security and Disaster Engineering of Ministry of Education, Beijing University of \\ Technology, Beijing 100124, China \\ * Corresponding author Email: gxc6307@163.com
}

\begin{abstract}
This paper presents a simple but workable modeling method to simulate the waveinduced liquefaction scenarios around a marine pipeline within the framework of the Biot's theory, incorporating the main features such as relation for the consolidation describing the porevolume reduction, hysteretic stress-strain behavior of soil skeleton and soil-pipe contact effect. In this context, special attention is paid to the implementation of a well-calibrated cyclic soil model for hysteretic and nonlinear stress-strain behavior (i.e. strain softening and cyclic degradation), associated with a semi-empirical shear-volume coupling equation for capturing the accumulative volumetric change, which links the increment of volumetric strain per cycle of wave with the shear strain occurring during that particular cycle. The proposed modeling framework is then incorporated into an explicit time matching finite difference analysis procedure, allowing a full non-linear dynamic analysis of the intensive interactions between the pipeline and the seabed undergoing buildup of pore pressure and residual liquefaction. Retrospective simulation of the wave flume test performed by Sumer et al. (2006c) using the proposed model shows good agreement, calibrating the reliability of the modeling method for the prediction of wave-induced liquefaction of sandy seabed and failure process of the buried pipelines. Finally, the liquefaction mechanism around a buried pipeline under a nonlinear wave loading is investigated by numerical examples. The obtained results interpret the cause of liquefaction and the resulting consequence for pipeline stability in wave environment.
\end{abstract}

Keywords: Wave-induced liquefaction; Submarine pipelines; Strain softening; Cyclic degradation; Pipeline-seabed interaction.

\section{List of symbols}

$\begin{array}{ll}A & \text { Davidenkov model parameter } \\ B & \text { Davidenkov model parameter } \\ C_{1} & \text { constant of shear-volume coupling model } \\ C_{2} & \text { constant of shear-volume coupling model } \\ C S S R & \text { cyclic shear stress ratio } \\ D & \text { pipeline diameter } \\ d & \text { water depth } \\ d_{50} & \text { grain size } \\ e & \text { distance from the center of the pipe to the mudline } \\ g & \text { body force acceleration } \\ G & \text { shear modulus }\end{array}$




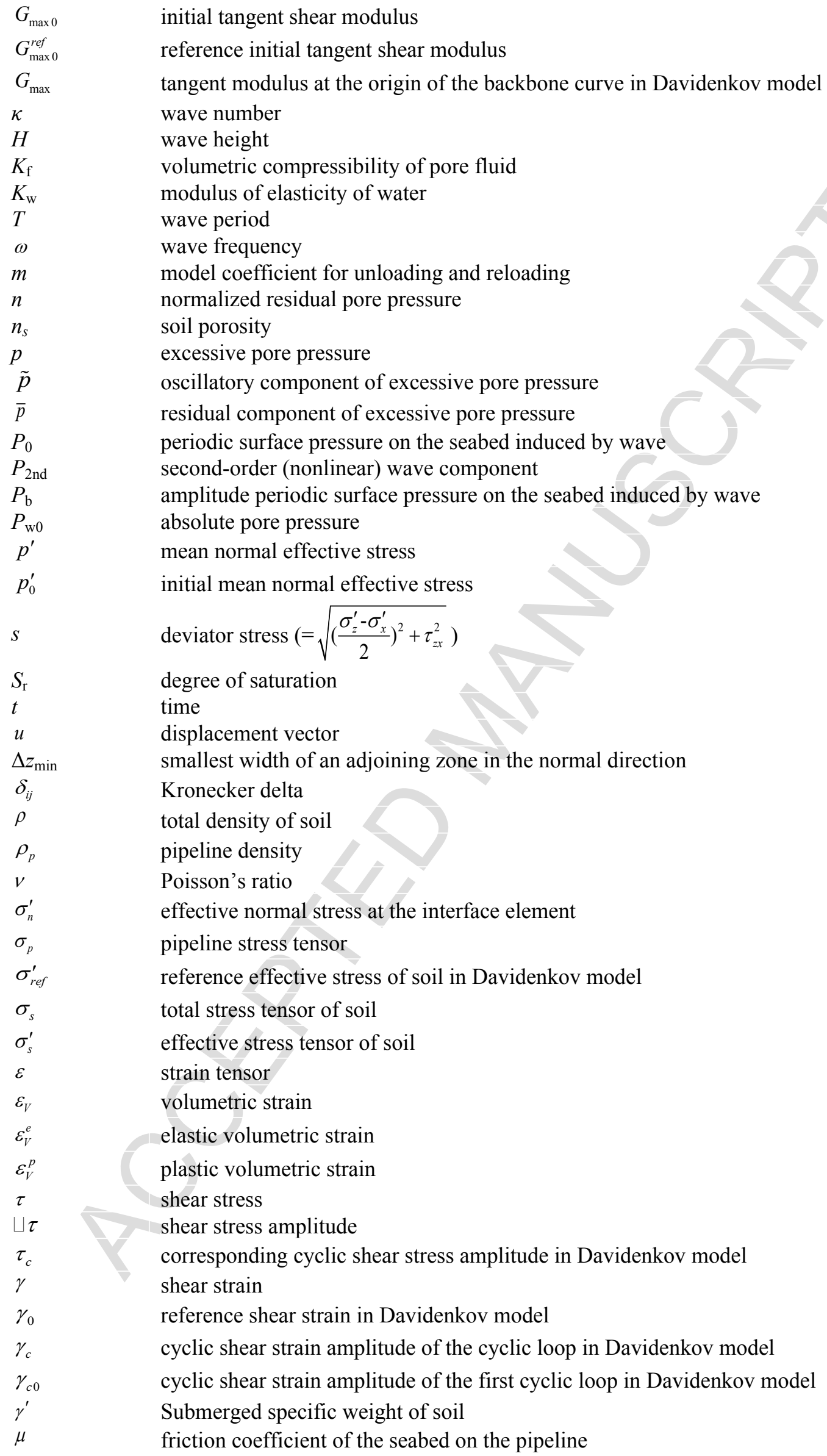


The stability of submarine pipelines buried in loose granular soils (e.g. for silt, fine sand and in some cases, gravel) is of major concern in practice. Failure of submarine pipelines has been observed to be linked to wave-induced instability of marine deposits leading up to liquefaction (de Groot et al., 2006; Fredsøe, 2016). The conventional pipeline stability concept (assuming the seabed is stable) may be challenged (or even violated) if the liquefaction-related sinking/floatation of the pipeline occurs (Palmer et al., 1996; 2004; Sumer et al., 2006a; Brennan et al., 2015). Particularly, liquefaction-induced instability of a pipeline primarily occurs in residual liquefied soil, which, in contrast to instantaneous liquefied soil, includes a larger area of the seabed in the vicinity of the pipeline simultaneously (Jeng et al., 2001; Jeng, 2013; Sumer, 2014). Therefore, it is vital to accurately evaluate both the wave-induced oscillatory and residual response of the liquefiable seabed around a pipeline for coastal engineers involved in the design of submarine pipelines.

The stability of a submarine pipeline involves complex interactions between the buried pipeline and the neighboring soil undergoing buildup of pore pressure and residual liquefaction. Recent developments in field and laboratory studies (e.g. centrifuge tests and wave flume modeling) combined with numerical modeling of the wave-induced response of submarine pipelines in the liquefied seabed have together brought about improvements in the understanding of the physics behind the dynamic pipeline-seabed interaction process (Teh et al., 2003; 2006; Sumer et al., 2006a; 2006b; 2006c; 2012; Kirca et al., 2013; 2014; Sassa and Sekiguchi, 1999; 2001; Sawicki and Mierczyński, 2006; Gao et al., 2011). The presence of pipeline in the soil has a major consequence for the response of the surrounding soil and over areas of contact with the loading boundaries, involving deformations, buildup of pore pressures and resulting liquefaction patterns (compared with those developing in homogeneous soil). This dynamic evolution of the pipe-soil interaction makes the liquefaction-related stability problem of a submarine pipeline fascinating to deal with, but also increases the complexity of an accurate model or description of the phenomena. To date, relatively few contributions are available in the literature for numerical models of liquefaction around a buried pipeline. The use of the Biot's theory framework to encapsulate the soil behavior incorporating the effects of the transient flow of the pore-fluid through the voids, offers an attractive alternate framework (Biot, 1941; 1956; Sawicki and Mierczyński, 2006; Small et al., 1976; Prèvost et al., 1976; Popescua et al., 2006). These Biot-type models are generally provided by finite element (FE)/finite difference element scheme in the time-domain, taking into account the solid-fluid interaction by means of a coupled effective stress formulation, more complex geometrical schematisations and the possible contact with the buried pipeline (Heider et al., 2014; Di and Sato, 2003; Tian et al., 2010; Luan et al., 2008; Ulker and Rahman, 2009). Among these, Zhao et al. (2014) and Zhao and Jeng (2016) developed a two-dimensional integrated numerical model to investigate the residual soil response in the vicinity of a buried pipeline and a partially-backfill-trenched pipeline, in which the phase-resolved oscillatory shear stress is used as a source for the build-up of residual pore pressure (Jeng and Zhao, 2015). Later in Zhao et al. (2016), the cross-anisotropic soil behavior is also included into the numerical model. However, the oscillatory and residual mechanisms are not coupled and simulated separately. Dunn et al. (2006) and Zhang et al. (2015) investigated the wave-induced liquefaction around the 
pipeline by using an advanced elasto-plastic soil model to couple oscillatory and residual response of liquefiable seabed and predicted both the possibilities of the residual and momentary liquefactions.

In all cases, the nonlinear soil behavior has a significant influence on the soil-structure interaction, precipitating concentration of strain and deformation towards the loading boundaries (imposed by the contact effects between soil and structure) (Bathe and Chaudhary, 1985; Jardine et al., 1986; Clayton, 2011). It is well known that soils are characterized by nonlinear stress-strain behaviour under cyclic shearing, involving strain softening and cyclic degradation (as described by, e.g. Vucetic and Dobry, 1991). Soils exhibit nonlinear behavior even at very small strain (Clayton, 2011). Strain softening refers to the behavior that soil stiffness may decay with strain by orders of magnitude and the corresponding increase in material damping with increasing shear strain level, while cyclic degradation represents the cycle-by-cycle degradation of saturated sand due to the buildup of pore pressure under cyclic shearing. Nevertheless, to the authors' best acknowledgement, no theoretical models are yet available in the literature for wave-induced liquefaction around a buried pipeline incorporating the cyclic nonlinearity of soil stiffness (i.e. strain softening and cyclic degradation).

The purpose of this paper is to develop a simple but workable modeling method to simulate the wave-induced liquefaction scenarios around a buried pipeline within the framework of the Biot's theory, incorporating the main features such as relation for the consolidation describing the porevolume reduction, nonlinear stress-strain behavior of soil skeleton and soil-pipe contact effect. In this context, special attention is paid to the implementation of a well-calibrated cyclic soil model for nonlinear and hysteretic stress-strain behavior, associated with a semi-empirical shear-volume coupling equation for capturing the plastic volumetric change, which links the increment of volumetric strain per cycle of wave with the shear strain occurring during that particular cycle. The proposed modeling method is then incorporated into an explicit time matching finite difference analysis procedure, allowing a full non-linear dynamic analysis of the intensive nonlinear interaction between the pipeline and liquefiable seabed. Retrospective simulation of the wave flume test by Sumer et al. (2006c) using the proposed model are performed to calibrate the reliability of the modeling method. Finally, the liquefaction mechanism around a buried pipeline under a nonlinear wave loading is investigated by numerical examples. The influence of nonlinear wave component upon the wave-pipeline-seabed interaction is also studied.

\section{Definition of the problem}

\subsection{Problem layout}

Fig. 1 shows schematically the problem we consider. The figure shows that a pipeline with an outer diameter $(D)$ is fully buried within a liquefiable seabed with finite thickness $(h)$. The wave, pipe and seabed models are integrated into one single model. To facilitate the interpretation of the results, the storm propagation along the porous seabed is simulated by using the Stokes wave theories. Generally, the assumption may be introduced here to clarify the most essential phenomena, satisfying the requirements of most engineering problems. (Gao et al. 2003).

As depicted in Fig. 1, the dynamic system consists of two sub-domains, the structure and the seabed (unbounded half-space). The propagation of ocean waves exerts significant dynamic 
loading on the seabed deposits. The porous seabed consequently responds to the wave loading and undergoes consolidation. This will result in pore-pressure generation and in some cases, liquefaction of the sandy seabed, therefore precipitating complex pipeline-seabed interaction. In this study, the problem of wave-induced interactions between pipeline and seabed is treated within the framework of Biot theory, allowing for both generation and partial dissipation of excess pore pressure during wave propagation. All the classic assumptions of Biot theory are admitted, only with exception of the seabed deposits being nonlinear and hysteretic under cyclic wave actions.

\subsection{Governing equations}

Before presenting the proposed modeling scheme, it is instructive to give a brief resume of the mathematical framework governing the dynamic process of pipeline-seabed system under prescribed boundary conditions (Zienkiewicz et al., 1980; Ulker et al., 2009).

The pipeline can be practically treated as an impermeable rigid medium. Based on an elastic theory, the governing equilibrium equations for buried pipeline can be expressed as

$$
\sigma_{\mathrm{p} i j, j}+\rho_{\mathrm{p}} g=0
$$

where $\sigma_{\mathrm{p}}=$ pipeline stress tensor; $\rho_{\mathrm{p}}=$ pipeline density; and $g=$ body force acceleration.

The saturated sand is modeled as a two-phase medium. The effective stress in the skeleton for a two-phase soil medium is defined by

$$
\sigma_{\mathrm{s} i j}^{\prime}=\sigma_{\mathrm{s} i j}+\delta_{i j} p
$$

where $\sigma_{\mathrm{s}}=$ total stress tensor of soil; $\sigma_{\mathrm{s}}^{\prime}=$ effective stress tensor of soil; $\delta_{i j}=$ Kronecker delta; and $p=$ excessive pore pressure.

It is well known that the wave-induced excess pore pressure can be separable into the following terms (as schematically illustrated in Fig. 2),

$$
p=\tilde{p}+\bar{p}, \quad \text { where } \bar{p}=\frac{1}{T} \int p d t
$$

where $T=$ wave period; $p=$ wave-induced excess pore pressure; $\tilde{p}=$ transient (oscillatory) component; and $\bar{p}=$ residual (buildup) component (which is the period-averaged value of excess pore pressure $p$ ).

Based on the Biot's consolidation equation, the governing equations for the force equilibrium in (nearly) saturated soil can be written as follows:

$$
G \nabla^{2} u_{i}+\left(\frac{G}{1-2 v}\right) \varepsilon_{j j, j}=p_{, i}+\rho \ddot{u}_{i}
$$

where $u=$ soil displacement vector; $\varepsilon=$ soil strain tensor; $\rho=$ total density; $p=$ excess pore pressure; $v=$ Poisson's ratio; and $G=$ shear modulus of soil. A saturated porous seabed is assumed to be hydraulically isotropic (i.e. the same permeability, $k$, in all directions) and obey Darcy's law. With these assumptions, the continuity of flow condition 
yields the following equation:

$$
\frac{k}{\gamma_{\mathrm{w}}} \nabla^{2} p-\dot{p} n_{\mathrm{s}} / K_{\mathrm{f}}=\dot{\varepsilon}_{j j}
$$

145

where $p=$ wave-induced excess pore pressure; $n_{\mathrm{s}}=$ soil porosity; $\gamma_{\mathrm{w}}=$ unit weight of water in the pore; and $K_{\mathrm{f}}=$ volumetric compressibility of pore fluid, which is a function of the modulus of elasticity of water $\left(K_{\mathrm{w}}\right.$, generally taken as $\left.2 \times 10^{9} \mathrm{~N} / \mathrm{m}^{2}\right)$, the degree of saturation $\left(S_{\mathrm{r}}\right)$ and the absolute pore pressure $\left(P_{\mathrm{w} 0}\right)$, defined as follows:

$$
\frac{1}{K_{\mathrm{f}}}=\frac{1}{K_{\mathrm{w}}}+\frac{1-S_{\mathrm{r}}}{P_{\mathrm{w} 0}}
$$

The term in the right-hand side of Eq. (5) is the increment of volumetric strain of soil subjected to cyclic loading, which can be decomposed to an elastic part ( $\left.d \varepsilon_{\mathrm{V}}^{\mathrm{e}}\right)$ and a plastic part (d $\left.\varepsilon_{\mathrm{V}}^{\text {p }}\right)$, i.e.

$$
\mathrm{d} \varepsilon_{\mathrm{V}}=\mathrm{d} \varepsilon_{\mathrm{v}}^{\mathrm{e}}+\mathrm{d} \varepsilon_{\mathrm{V}}^{\mathrm{p}}
$$

In particular, $\mathrm{d} \varepsilon_{\mathrm{v}}^{\mathrm{p}}$ is defined as the volumetric strain increment to represent the compaction of the skeleton configuration due to cyclic shear strains, which gradually rearrange the soil grains at the expense of pore volume of soil, causing associated instances of pore-pressure accumulation $\bar{p}$ (Kirca et al., 2013).

The domains of pipeline and seabed are joined by an interface, which will be discussed in detail in the following section.

\section{Cyclic soil constitutive model}

The accuracy of predictions of wave-pipe-seabed interactions hinges on realistically modeling soil behavior incorporating cyclic loading effects. From extensive evidence of laboratory and field tests, it is recognized that sandy soils behave highly nonlinear (even at very small strain), hysteretic (absorbing energy solely as a function of cyclic strain) and nearly always contraction or densification, subjecting to cyclic shearing.

In this section, a pragmatic constitutive model is present, separating the basic stress-strain behavior of soil skeleton and the accumulative volume compaction (Zienkiewicz et al., 1978; Sawicki and Mierczyński, 2006). The concept of autogenous volumetric strain using a semiempirical shear-volume coupling equation (Sect. 3.2) is introduced, coupled with a well-calibrated hysteretic stress-strain model (Sect. 3.1).

\subsection{Cyclic soil stress-strain relation}

The well-calibrated Davidenkov model is used to describe the hysteretic damping coupled with nonlinear stress-strain characteristics of granular soil skeleton, in conjunction with Masing rules. The cyclic soil model is characterized by a backbone curve and rules that describe unloadingreloading behavior and cyclic modulus degradation, as illustrated in Fig. 3 (Matasović and Vucetic, 1993; Stewart et al., 2008). The shear modulus and damping ratio are the controlling 
factors to determine the model parameters, which strongly depend on the cyclic shear strain and the effective stress state (or the residual pore pressure). Within the framework of the Biot theory, the aforementioned governing equations can be easily generalized to the cyclic material behavior if the stress-strain relation is written incrementally (Small et al., 1976; Zienkiewicz et al., 1980; Borja et al., 1998).

According to Martin and Seed (1982), the Davidenkov constitutive model gives the shear stress in terms of an explicit function of the shear strain, which is more convenient in the cyclic straincontrolled approach for the evaluation of dynamic response, as follows,

$$
\tau=G \cdot \gamma=G_{\max } \cdot \gamma \cdot[1-H(\gamma)]
$$

Among which, $H$ is a function that describes the basic shape of shear-stress-shear-strain relationship. Specifically in the Davidenkov form, the soil behavior is constructed using a hyperbola to represent the shape of stress-shear curve as follows,

$$
H(\gamma)=\left\{\frac{\left(\gamma / \gamma_{0}\right)^{2 B}}{1+\left(\gamma / \gamma_{0}\right)^{2 B}}\right\}^{A}
$$

where $\tau=$ shear stress; $\gamma=$ shear strain; $G_{\max }=$ initial tangent shear modulus (at the origin of initial backbone curve); $A, B$ and $\gamma_{0}$ are experimental parameters.

Taking the derivatives of $\gamma$ in Eq. (8), time-variant tangent shear modulus of initial backbone curve is obtained as follows,

$$
G^{t+\Delta t}=\frac{d \tau}{d \gamma}=G_{\max }\left[1-\left(1+\frac{2 A B \gamma_{0}^{2 B}}{\gamma_{0}^{2 B}+\gamma^{2 B}}\right) \cdot H(\gamma)\right]
$$

The initial backbone curve coincides with the initial monotonic stress-strain curve. To construct the hysteresis curve, we employ the dynamic backbone curve in association with the Masing rules to describe the unloading and reloading branches of cyclic loops. This is particularly true for the soil element subject to progressive wave loading with constant amplitude (Pyke, 1979). That is,

$$
\tau-\tau_{\mathrm{c}}=G_{\max } \cdot\left(\gamma-\gamma_{\mathrm{c}}\right) \cdot\left[1-H\left(\frac{\left|\gamma-\gamma_{\mathrm{c}}\right|}{2 m}\right)\right]
$$

where $\gamma_{\mathrm{c}}=$ cyclic shear strain amplitude of a particular cyclic loop; $\tau_{c}=$ corresponding cyclic shear stress amplitude; and the coefficient $m=1$ for unloading and reloading, according to Masing criteria.

Taking the derivatives of $\left(\gamma-\gamma_{c}\right)$ in Eq. (11), time-variant tangent shear modulus of hysteresis curves is thus obtained as follows,

$$
\begin{aligned}
& G^{t+\Delta t}=\frac{d\left(\tau-\tau_{\mathrm{c}}\right)}{d\left(\gamma-\gamma_{\mathrm{c}}\right)} \\
& =G_{\max }\left\{1-\left[1+\frac{2 A B\left(2 \gamma_{0}\right)^{2 B}}{\left(2 \gamma_{0}\right)^{2 B}+\left|\gamma-\gamma_{\mathrm{c}}\right|^{2 B}}\right] H\left(\frac{\left|\gamma-\gamma_{\mathrm{c}}\right|}{2}\right)\right\}
\end{aligned}
$$

Note that the octahedral or generalized shear stress and strain are adopted in the abovementioned equations for 2D or 3D nonlinear dynamic problems (Huo et al., 2004). The tensor equation of equivalent shear strain algorithm as an incremental form based on the second invariant of the 
201

strain deviator tensor $J_{2}$ can be expressed as

$$
\gamma^{t+\Delta t}=\gamma^{t}+\operatorname{sign} \cdot\left|\Delta \gamma^{t+\Delta t}\right|
$$

202

in which

$$
\gamma=2 \sqrt{\frac{2}{3} J_{2}}
$$

At each time step, the incremental shear strain $\Delta \gamma^{t+\Delta t}$ is monitored to identify the loading and unloading branches of the stress-strain loop. The sign of $\Delta \gamma^{t+\Delta t}$ is positive for reloading and negative for unloading. Once the stress-strain curve enters into the next cycle from the stress reversal point as illustrated in Fig. 2, the corresponding $\gamma_{c}$ of the particular cyclic loop is updated and assigned by the shear strain at the reversal point.

In reality, the initial tangent shear modulus $G_{\max 0}$ of soil generally varies strongly with the mean normal effective stress in the sandy seabed. In the first loading cycle, we assume the following equation to consider this effect, according to normal geotechnical practice (Jeng, 2001; de Groot et al., 2006),

$$
G_{\max 0}=G_{\max 0}^{\mathrm{ref}} \sqrt{\frac{p_{0}^{\prime}}{\sigma_{\text {ref }}^{\prime}}}
$$

where $\sigma_{\text {ref }}^{\prime}=$ reference effective stress (taken as $100 \mathrm{kPa}$ in this study); $G_{\max 0}^{\text {ref }}=$ reference initial tangent shear modulus; and $p_{0}^{\prime}=$ initial mean normal effective stress, defined as

$$
p_{0}^{\prime}=\gamma^{\prime} z \frac{1+2 K_{0}}{3}
$$

where $z=$ vertical distance measured from the mudline downward; $\gamma^{\prime}=$ submerged specific weight of soil; and $K_{0}=$ coefficient of lateral earth pressure.

In the second and subsequent wave cycles, the cyclic shearing causes gradual buildup of pore pressure and subsequent reduction of the mean normal effective stresses in the soil skeleton. Consequently, the tangent modulus $G_{\max }$ at the origin of the corresponding backbone curve degrades. The modulus degradation model (Hardin and Drnevich, 1972) is therefore employed to simulate the cyclic softening phenomenon, with the residual pore pressure as the degradation parameter.

$$
G_{\max }=G_{\max 0} \sqrt{\frac{\sigma_{0}^{\prime}-\bar{p}}{\sigma_{0}^{\prime}}}=G_{\max 0} \sqrt{1-n}
$$

222 where $n=\bar{p} / \sigma_{0}^{\prime}$ is normalized residual pore pressure; $\sigma_{0}^{\prime}=$ initial mean normal effective stress.

It is noted that during a shear loading process near liquefaction (the normalized residual pore pressure reaches 1 , that is, $n=1$ ), the saturated sand exhibits a dilative tendency increases effective confinement, allowing the soil to develop some stiffness and strength (Elgamal et al., 2003). Generally, the amount of tangent shear modulus $G_{\max }$ is set equal to $0.01 G_{\max 0}$ when liquefaction occurs at that particular cycle. 


\subsection{Evaluation of plastic volumetric strain}

The essential part of the cyclic soil constitutive model is the development of a suitable model for the autogenous volumetric compaction, which is the major controlling factor of the pore-pressure accumulation. To this end, a separate expression (with respect to the constitutive model above) is proposed in this section to determine the progressive increase of the volumetric strain due to the compaction of grain configuration under cyclic shearing.

The accumulated volumetric strain is mainly a consequence of the cyclic shear strain induced by the action of repeated wave loading. Martin et al. (1975) presented quantitative data to illustrate the shear-volume coupling mechanism and showed that the amount of compaction per cycle was proportional to the cyclic shear strain amplitude and accumulated volume compaction and is independent of normal effective stress. Subsequently, Byrne (1991) simplified this equation and recommended an incremental volumetric strain $\left(\Delta \varepsilon_{\mathrm{V}}^{\mathrm{p}}\right)$ definition with only two parameters as follows,

$$
\frac{\Delta \varepsilon_{\mathrm{V}}^{\mathrm{p}}}{\gamma_{\mathrm{c}}}=C_{1} \exp \left(-C_{2}\left(\frac{\varepsilon_{\mathrm{V}}^{\mathrm{p}}}{\gamma_{\mathrm{c}}}\right)\right)
$$

where $\gamma_{\mathrm{c}}=$ amplitude of cyclic shear strain in percent induced by the wave-induced cyclic shear stress $; \varepsilon_{\mathrm{V}}^{\mathrm{p}}=$ accumulated volumetric strain from previous cycles in percent; and $C_{1}, C_{2}=$ model constants for the soil in question.

The macroscopic coefficients $C_{1}, C_{2}$ take their physical meaning in part from the microscopic characteristics of the medium, which can be easily calibrated from laboratory testing. Byrne (1991) model is based on physical laws and back-fitted extensive experimental data. Particularly, one can easily incorporate this equation into the proposed procedure using the cyclic soil stress-strain relation mentioned above.

\section{Numerical scheme}

In this section, we introduce a relatively simple procedure based on effective stress concepts which will permit nonlinear analysis taking account of dynamic pipeline-seabed interaction to be realistically carried out, especially when the wave-induced liquefaction scenario is considered. The dynamic analysis procedure involves a simulation of a pipeline buried in the liquefiable seabed, including modeling of cyclic soil behavior (Sect 4.2), nonlinear pipeline-seabed contact (Sect 4.2), hydrodynamic forces, and boundary conditions (Sect. 4.3).

\subsection{Computation domain}

The computation domain should be large enough so that the external boundary can represent an infinitely extended medium. Otherwise, the presence of the artificial boundaries will induce a significant influence on the response of the seabed in the vicinity of pipeline. A rule-of-thumb is that the grid in the lateral boundary is built by an expansion factor of 5 in relation to the wave length $L$. In this study, a value of approximately $5 L$ is considered as satisfactory.

The computation domain is discretized into a set of structured grid zones. The elements used are four-noded, constant-strain quadrilateral finite difference elements, with the internal angles close 
to $90^{\circ}$, the aspect ratios close to 1 . As shown in Fig. 4, a trade-off with the high computational time and restriction of hardware resources has to be found. In the vicinity of pipeline, a high level of mesh refinement is desirable to capture high strain gradients, as the accuracy of the simulation depends on the ability of the grid layout to represent such gradients. Therefore, in this study, a very refined region around the pipeline and a coarser zone away from it has been used as shown in Fig. 4.

\subsection{Pipeline-soil contact simulation}

Complex contact can occur between the pipeline and surrounding seabed during wave propagation. Significant changes in the contact region are possible including relative sliding with Coulomb friction or even possible separation, which is unknown prior to the analysis. In this section, zero-thickness interface elements are used to represent this boundary nonlinear behavior, which is characterized by normal and shear stiffness, and sliding properties.

With respect to the interface element, no-overlapping occurs between the pipeline and the support seabed and no slip before yielding of the interface is assumed, the stiffnesses are actually penalty numbers that approximately enforce contact-surface compatibility consisting of impenetrability and pre-sliding stick constraints. The normal and shear stiffnesses are therefore set to be much greater than the stiffness of the softer neighbouring zone. A rule-of-thumb rule in the FLAC manual (Itasca, 2006) is that the normal and shear stiffness can be set equal to ten times the equivalent stiffness of the softer neighbouring zone, which is given by:

$$
\frac{K+4 / 3 G}{\Delta z_{\min }}
$$

where $K$ and $G$ are the bulk and shear moduli, respectively, and $\Delta z_{\min }$ is the smallest width of an adjoining zone in the normal direction.

It is to note that the shear sliding only occurs when the shear stress $\tau$ at the interface element is greater than frictional resistance, which is defined by Coulomb friction law.

$$
|\tau| \geq \sigma_{\mathrm{n}}^{\prime} \mu
$$

where $\sigma_{\mathrm{n}}^{\prime}=$ effective normal stress at the interface element; and $\mu=$ friction coefficient of the seabed on the pipeline, taken as 0.7 in this study (Luan et al., 2008).

No dilation of the interface element is assumed after yielding (i.e. sliding).

\subsection{Boundary conditions}

Essentially, appropriate boundary conditions are necessary to specify the complex wave-seabedpipeline interaction problem.

As mentioned earlier, the wave actions follow the Stokes wave theories. The second-order approximation of non-linear wave pressure in two-dimensional problems is applied as periodic surface pressure at the seabed surface $P$ (Gao et al. 2003; Gao and $\mathrm{Wu}, 2006$ ), as follows:

$$
P=P_{\mathrm{b}} \cos (\kappa x-\omega t)+P_{2 \mathrm{nd}}
$$

where $P_{\mathrm{b}}=\frac{\gamma_{\mathrm{w}} H}{2 \cosh \kappa d}$ is the amplitude of the first-order (linear) wave pressure, $P_{2 \mathrm{nd}}$ is the secondorder (nonlinear) wave component which is given by 
298

$P_{2 \text { nd }}=\left(\frac{\kappa H}{2}\right)^{2} \frac{\gamma_{\mathrm{w}}}{\kappa}\left[A_{21}+A_{22} \cos 2(\kappa x-\omega t)\right]$

$A_{21}=-\omega_{0} \beta_{1}-\frac{\omega_{0}^{2}}{4 \sinh ^{2} \kappa d}, A_{22}=4 \omega_{0} \beta_{2}-\frac{\omega_{0}^{2}}{4 \sinh ^{2} \kappa d}, \omega_{0}=\sqrt{\tanh \kappa d}, \beta_{1}=\frac{1}{8}\left(-\omega_{0}^{-3}+\omega_{0}\right)$

$\beta_{2}=\frac{3\left(\omega_{0}^{-7}+\omega_{0}\right)}{16} \cosh (2 \kappa d)$

where $\kappa=$ wave number, $\omega=$ wave frequency, $H=$ wave height, and $d=$ water depth.

The wave-induced shear stress at the seabed surface has negligible influence on the soil shear stress compared with the stresses induced by the surface pressure from the waves (Sawaragi and Deguchi, 1992).

$$
\sigma_{\mathrm{zz}}^{\prime}=\sigma_{\mathrm{zx}}^{\prime}=0 \text { at } z=0
$$

Furthermore, the boundary should also satisfy the following conditions:

- Free draining boundary at the top of the seabed is remained.

- For the impermeable rigid bottom boundary, the dynamic fluctuations of all the physical quantities vanish, i.e.

$$
u_{\mathrm{x}}=0, u_{\mathrm{z}}=0, \partial p / \partial z=0, \text { at } z=H
$$

- As the computation domain is large enough that lateral boundaries have no influence on the pipeline-seabed interactions, both lateral boundaries of the seabed are therefore fixed.

Finally, no flux flow can occur at the interface between the pipeline and surrounding seabed, due to the impermeable assumption of pipeline. Consequently, the pore gradient at the outer surface of the pipeline should vanish, i.e.

$$
\partial p / \partial n=0, \text { at } r=\sqrt{\left(x-x_{0}\right)^{2}+\left(z-z_{0}\right)^{2}}=D / 2
$$

where $D=$ diameter of pipeline.

\subsection{Analysis procedure}

The proposed analysis framework using the dynamic coupled stress-flow finite difference scheme is developed within the Fast Lagrangian Analysis of Continua (FLAC3D) 3.0 code environment (Itasca, 2002). The cyclic soil model associated with the shear-volume coupling equation is compiled by the $\mathrm{C}++$ computer language and implanted into the FLAC3D code to realistically capture the dynamic response of the seabed. Furthermore, the Biot consolidation equation, Darcy hydraulic flow and pore fluid stiffness are basic to the FLAC3D code. The proposed modeling method is then incorporated into an explicit time matching finite difference analysis procedure, in which the aforementioned governing equations are satisfied by employing a sufficient small time step $\Delta t$ (in this study, $\Delta t=10^{-5} \mathrm{~s}$ ).

The initial consolidation status of seabed is essential to simulate the natural marine environments. Prior to the wave-induced liquefaction analysis, static analysis is performed to set up the initial 
stress state of the seabed model in the vicinity of the pipeline. We apply pore water pressure and self-weight of soil deposits at each node, and then release the freedom of pore water pressure in order to obtain the initial effective stresses within the seabed.

\subsection{Model validation}

It is the experiment that decides as to the value of a particular theoretical model, especially in engineering sciences. In this section, the validation of the present model is carried out against i) a set of cyclic loading tests on the natural Nanjing fine sand with relative density $D_{\mathrm{r}}$ of $50 \%$, and ii) the wave flume test performed by Sumer et al. (2006c), respectively.

With respect to the response of single elements of soil, undrained 2D loading of a soil element is considered. Note that only a single element is modeled to avoid the discretisation error as the finite difference program (i.e. FLAC3D code) only integrates the constitutive model over the prescribed loading path. Incorporating the complex loading boundaries imposed by pipe-seabed interaction, undrained shear with the continuous rotations of principal stress axes is applied in conjunction with cyclic change in the deviator stress $q\left(=\sqrt{\left(\frac{\sigma_{\mathrm{z}}^{\prime}-\sigma_{\mathrm{x}}^{\prime}}{2}\right)^{2}+\tau_{\mathrm{zx}}^{2}}\right)$. The shearing conditions essentially correspond to the undrained triaxial torsional tests of Chen et al. (2016), as graphically shown in Fig. 5 (i.e. the loading path is an ellipse in $\tau_{\mathrm{zx}}-\left(\frac{\sigma_{\mathrm{z}}^{\prime}-\sigma_{\mathrm{x}}^{\prime}}{2}\right)$ space). The constitutive parameters used are listed in Table 1.

As plotted in Fig. 6, the predicted soil behaviour conforms well to the experimentally observed soil behavior at a wide strain range. However, when the soil approaches the liquefied state (i.e. $n=1$ ), the present model tends to deviate slightly from the observed stress-strain loops. This might be traced back to the cyclic mobility phenomenon of sandy soils close to and during liquefaction, which results in significant regain in soil shear stiffness and strength (Elgamal et al., 2003). Due to the inability to capture the cyclic mobility, conservative results of soil responses (including pore pressure and effective stress) might be obtained by the present model. Generally, the characteristic post-liquefaction response (i.e. amplitude and trend of stress-strain loops) can be captured by the present constitutive model. Specifically, a more sophisticated volume-shear coupling model is needed for accurate characterization of post-liquefied state.

Sumer et al. (2006c) conducted an experimental study for the pore pressure accumulation and liquefaction in liquefiable seabed under progressive waves. The model seabed was consisted of silt (with $d_{50}=0.045 \mathrm{~mm}$ ) placed in a $0.17 \mathrm{~m}$ deep and $0.9 \mathrm{~m}$ long box of the testing wave flume. An 8-cm pipe was installed with its center $e=12.5 \mathrm{~cm}$ below the seabed surface, which was exposed to progressive waves and became liquefied. The real-time monitor of pore-water pressure was carried out in the far field and around the pipe simultaneously.

This wave flume test is numerically simulated, using the proposed modeling method. The input parameters are tabulated in Table 2. Fig. 7 shows the time series of the excess pore pressure at two locations: at the bottom of the pipe; and in the far field at the same level as the pipe bottom from the present model and Sumer et al. (2006c). It is seen that the overall trend of the excessive pore pressure of the present model captures the experimental data well. However, the oscillatory pore pressure amplitude of the present model is relatively greater than that from the experiment. The discrepancy might be traced back to the fact that the small-scale experiment has scale effect on the response of excessive pore pressure, while the numerical model cannot consider this effect. As far 
as the residual pore pressure is concerned, very satisfactory identification can be found in the present model. This comparison demonstrates the accuracy of the proposed model in predicting the wave-induced dynamic response of the seabed in the vicinity of pipeline.

\section{Numerical examples}

To gain a better understanding of wave-induced liquefaction around a buried pipeline, the dynamic process of pipe-seabed interactions will be analyzed by means of numerical examples for the hypothetical case of a pipe with diameter of $2 \mathrm{~m}$ installed with its center $1.5 \mathrm{~m}$ below the seabed surface. A silty seabed is concerned with relative density $D_{\mathrm{r}}$ of $50 \%$. The constitutive parameters are calibrated from the shear stiffness and damping ratio decay curves of a natural marine silt (as shown in Fig. 8). The input parameters are specified in Table 3.

In real ocean environments, ocean waves are generally characterized as nonlinear. Special attention is therefore paid to the investigations in detail of the influence of nonlinear wave component upon the wave-pipeline-seabed interaction (Sect. 5.1), the phenomena occurring close to the pipeline-seabed interface under nonlinear wave loadings (Sect. 5.2) and of the explanations for causes and consequences of nonlinear wave-induced liquefaction around a pipeline (Sect. 5.3).

\subsection{Effect of the nonlinear component of wave loading}

Fig. 9 plots the distributions of the wave-induced pore pressure versus time $(t)$ under wave loadings with and without the nonlinear component. As shown in the figure, the pore pressures along the perimeter of the pipe (i.e. top, side edge and bottom) buildup faster for the case under a nonlinear wave loading. For example, at the side edge of the pipeline, the accumulation of the pore pressure occurs intensively and reaches the initial mean normal effective stress (i.e. liquefaction initiation) at the $26^{\text {th }}$ wave cycle, as compared to the $40^{\text {th }}$ wave cycle for the case under linear wave loading. In addition, the influence of nonlinear wave component upon the soil response at the side edge of pipeline is more significant than those at the top and bottom of pipeline.

Fig. 10 further compares the liquefaction process of seabed under waves with and without the nonlinear component. As expected, the liquefied zone under a nonlinear wave is greater than that under a linear wave. It is to note that the liquefaction rate is also changed due to the existence of the nonlinear wave component. Therefore, combining Fig. 9 and Fig. 10, we can conclude that the wave non-linearity can not be always ignorable, especially upon the soil responses around a buried pipeline.

\subsection{Progressive liquefaction of pipeline-seabed system}

Fig. 10 also schematically shows the liquefaction process of the pipeline-seabed system under continuous wave loadings. It follows from the results shown in the figure that different progressive patterns are observed with reference to the nature of the liquefaction zone in the farfield regime and the near-field regime. At the far field, liquefaction initially occurs at the very top of the seabed, and gradually advances downwards. The nature of the liquefaction zone is indeed 1D pattern along the depth of seabed. The 1Dpattern travels with the wave and reaches a constant value (in the case under nonlinear wave loading, $2.2 \mathrm{~m}$ ) after 40 wave cycles. Unlike the 
progressive manner at the far field, the liquefaction initiates, and localizes significantly at the bottom of the pipe. Since then, the liquefaction spreads out quickly along the perimeter of the pipe in the upward direction and progressively enters the area of liquefaction at the far field. It is to note that the maximum liquefaction depth at the bottom of the pipeline reaches a significant amount of $4.2 \mathrm{~m}$ under a nonlinear wave, as compared to $2.2 \mathrm{~m}$ at the far field.

The residual liquefaction failure of the soil is due to the accumulation of pore pressure. Fig. 11 plots the entire buildup processes of pore pressure along the perimeter of the pipe (i.e. top, side edge and bottom) and in the far field at the corresponding positions (at the same level) in the case with the nonlinear wave component. It is seen that the excess pore pressure at the top and side edge of pipe accumulates practically the same as the corresponding far-field pressures. In contrast, the discrepancy becomes apparent on the excess pore pressure at the bottom of the pipe and that in the far field at the same level. At the bottom of the pipe, the accumulation of the pore pressure occurs intensively at the first several cycles (in this case, the initial 8 wave cycles), and subsequently reaches the initial mean normal effective stress (i.e. liquefaction initiation). One can clearly see that the oscillatory pore pressure amplitude is significantly amplified at the bottom of the pipe during the course of the buildup of excess pore pressure. This is due to the fact that the present model incorporates the effects of residual pore pressure on the degradation of the soil stiffness, and in turn on the amplification of the oscillatory component of excessive pore pressure. The progressive liquefaction of the seabed-pipeline system can also be clearly seen in the effective-stress path of the soils representatively at the bottom of the pipeline and at the corresponding position in the far field, as shown in Fig. 12. Fig. 12 illustrates that the mean normal effective stress $p^{\prime}\left(=\frac{\sigma_{z}^{\prime}+\sigma_{x}^{\prime}}{2}\right)$ follows a general trend of continuous decrease towards the zero stress state at the far-field soils. The fluctuations of the deviator stress $q\left(=\sqrt{\left(\frac{\sigma_{z}^{\prime}-\sigma_{x}^{\prime}}{2}\right)^{2}+\tau_{z x}^{2}}\right)$ are generally not significant during the wave actions. More complicated situations occur in the vicinity of the pipeline. The amplitude of the cyclic shear stress progressively reduced in accordance with decreasing the mean normal effective stress. In fact, the effective stress does not monotonically decrease the same as the far-field condition. Significant oscillation can be observed after first 10 wave cycles or so. The associated shear stress decreases dramatically, and eventually leads to a state of $\sigma_{z}^{\prime}=\sigma_{x}^{\prime}$ (indicating the initiation of liquefaction).

\subsection{Wave-induced interactions between pipeline and seabed}

Laboratory evidences indicate that the stress conditions are the fundamental factors that affect the observed liquefaction susceptibility of saturated sands. In this section, emphasis will be placed on interpreting the loading characteristics (i.e. initial stress state and cyclic shear stress path) of the near-field soils induced by the intensive pipeline-seabed interactions under a nonlinear wave.

Fig. 13 displays the distribution of the vertical effective stresses of the seabed after primary consolidation under the gravitational forces. It is seen that the presence of the structure apparently changes the stress state at the near-field seabed. A significant stress relief at the bottom of the pipeline occurs during the consolidation process, which is the result of replacement of the soil by the pipe. Consequently, this improves the susceptibility to liquefaction near the bottom, with regard to that in the undisturbed region.

Fig. 14 shows the variation of the wave-induced shear stress in the seabed in terms of the torsional 
448 stress $\tau_{\mathrm{xz}}$ versus the stress difference $\frac{\sigma_{\mathrm{z}}^{\prime}-\sigma_{\mathrm{x}}^{\prime}}{2}$. As plotted, the cyclic shear stress at the far field

follows the typical paths with continuous principal stress rotation at a constant deviator stress in the stress space of normal stress difference and torsional shear stress as documented by Ishihara (1983). Nevertheless, the stress path at the pipe bottom is close to a skewed ellipse shape in the stress space, which implies that the rotation of the principal stress continues in association of significant change in the deviator stress. Note that the amplitude of the deviator stress is multiplied from $6 \mathrm{kPa}$ at the far field to $8.14 \mathrm{kPa}$ in this case.

Furthermore, the imposition of initial shear stress condition can be clearly seen in the stress path. In reality, the Cyclic Shear Stress Ratio (CSSR) is generally used as an index for evaluating the susceptibility to liquefaction of sands under cyclic loading (Ishihara, 1993). Seed and Rahman (1978) recognized that the CSSR is the dominant factor affecting the wave-induced residual liquefaction process, which is defined as

$$
\operatorname{CSSR}=\Delta \tau / p_{0}^{\prime}
$$

where $\Delta \tau=$ shear stress amplitude, $p_{0}^{\prime}=$ initial mean normal effective stress.

Combining Fig. 13 and Fig. 14, one can see that the CSSR at the pipe bottom is significantly amplified from that at the far field as a consequence of the intensive pipeline-seabed interactions. This can explain the influence of the pipeline on the buildup pattern of pore pressure and the resulting liquefaction in the vicinity of the structure.

Finally, the pipeline-seabed interactions can be comprehensively reflected in the stress-strain response of the soils at the near field. As plotted in Fig. 15, it is to note that high amplitudes of strain concentrate around the close neighbourhood of the structure. Liquefaction results in nearly $0.46 \%$ deviatoric strain in soils underneath the structure, while only $0.1 \%$ deviatoric strain is developed in the far-field soils at the same level (which are indeed not liquefied). Furthermore, significant degradation of the soil stiffness at the bottom of the pipeline is also observed, due to the rapid accumulation of pore pressure.

\section{Conclusions}

In this study, a simple but workable modeling method is proposed to simulate the wave-induced liquefaction scenarios around a pipeline within the framework of the Biot's theory, incorporating the main features such as relation for the consolidation describing the pore-volume reduction, nonlinear stress-strain behavior of soil skeleton and soil-pipe contact effect. In this context, we comprise the implementation of a well-calibrated cyclic soil model for nonlinear and hysteretic stress-strain behavior, coupled with a semi-empirical shear-volume coupling equation for capturing the plastic volumetric change. The proposed modeling method is then implemented into an explicit time matching finite difference analysis procedure and demonstrated with a quality wave flume test. From the analysis presented in this paper, the following conclusions can be drawn:

1. The main features characterizing the liquefaction process around a pipeline are incorporated into the proposed model. Retrospective simulation of the wave flume test performed by Sumer et al. (2006c) using the proposed model show good agreement, calibrating the reliability of the 
modeling method for the prediction of wave-induced liquefaction of seabed and failure process of the buried pipeline.

2. The numerical results indicate that the effects of wave nonlinearity on pore pressure and liquefaction along a buried pipeline can not be always ignored without significant errors, especially in association with the nonlinear soil behavior.

3. In the vicinity of the pipeline, the liquefaction process has a different progressive manner. Unlike at the far field, the liquefaction initiates, and localizes significantly at the bottom of the pipe. Since then, the liquefaction spreads out quickly along the perimeter of the pipe in the upward direction and progressively enters the area of liquefaction at the far field.

4. The intensive pipeline-seabed interactions impose complex loading boundaries at the interface between the pipeline and the surrounding soil. The Cyclic Shear Stress Ratio (CSSR) in the vicinity of the pipeline is amplified with respect to that at the far field, which is generally used as an index for evaluating the susceptibility to liquefaction of sands.

5. The nonlinear soil behavior has a significant influence on the soil-structure interaction, precipitating concentration of strain towards the loading boundaries. High amplitudes of strain concentrate around the close neighbourhood of the structure, leading to a rapid accumulation of pore pressure and resulting liquefaction.

\section{Acknowledgements}

The study in this paper was supported by the Projects of the National Natural Science Foundation of China (NSFC) (Grant No. 51608267; 51438004), and the National Key Basic Research Program of China (Grant No. 2011CB013605). This financial support is highly appreciated.

\section{References}

Bathe K J, Chaudhary A (1985) Solution method for planar and axisymmetric contact problems. Int J Numer Meth Eng, 21: 65-88.

Biot M A (1941) General theory of three dimensional consolidation. J Appl Phys, 12: 155-164.

Biot M A (1956) Theory of propagation of elastic waves in a fluid saturated porous solid, part I: Low frequency range. J Acoust Soc Am, 28(2): 168-177.

Borja R I, Tamagnini C, Alarcon E (1998) Elastoplastic consolidation at finite strain, Part 2: Finite element implementation and numerical examples. Comput Methods Appl Mech Engrg, 159(1-2): 103-122.

Brennan A, Sumer B M, Christensen E D, Kirca Ö, Sawicki A, Swidzinski W, Christensen E D. 386 (2015) D5.4 Guidelines for interaction between seabed and support structure. Deliverable 5.4 of the MERMAID Project, www.mermaidproject.eu.

Byrne P M (1991) A cyclic shear-volume coupling and pore pressure model for sand. In Proceedings 2nd International Conference on Recent Advances in Geotechnical Earthquake Engineering and Soil Dynamics, St. Louis, Missouri, USA: 47-55.

Byrne P M, Park S S, Beaty M, Sharp M, Gonzalez L, Abdoun T (2004) Numerical modeling of liquefaction and comparison with centrifuge tests. Can Geotech J, 41: 193-211.

Chen G X, Zhou Z L, Pan H, Sun T, Li X J (2016) The influence of undrained cyclic loading patterns and consolidation states on the deformation features of saturated fine sand over a wide strain range. Engineering Geology, 204: 77-93. 
Clayton C R I (2011) Stiffness at small strain: research and practice. Géotechnique, 61(1): 5-37.

Di Y, Sato T (2003) Liquefaction analysis of saturated soils taking into account variation in porosity and permeability with large deformation. Comput Geotech, 30: 623-635.

Dunn S L, Vun P L, Chan A H C, Damgaard J S (2006) Numerical modelling of wave-induced liquefaction around pipelines. J Waterw Port Coast Ocean Eng, ASCE, 132: 276-88.

de Groot M B, Kudella M, Meijers P, Oumeraci H (2006) Liquefaction phenomena underneath marine gravity structures subjected to wave loads. J Waterw Port Coast Ocean Eng, ASCE, 132 (4): $325-335$.

Elgamal A, Yang Z, Parra E, Ragheb A (2003) Modeling of cyclic mobility in saturated cohesionless soils. Int J Plast, 19(6): 883-905.

Fredsøe J (2016) Pipeline-seabed interaction. J Waterw Port Coast Ocean Eng, ASCE, 142(6): 03116002 .

Gao F P, Jeng D S, Sekiguchi H (2003) Numerical study on the interaction between non-linear wave, buried pipeline and non-homogeneous porous seabed. Comput Geotech, 30: 535-547.

Gao F P, Wu Y X (2006) Non-linear Wave Induced Transient Response of Soil around a Trenched Pipeline. Ocean Eng, 33: 311-330.

Gao F P, Yan S, Yang B, Luo C (2011) Steady flow-induced instability of a partially embedded pipeline: Pipe-soil interaction mechanism. Ocean Eng, 38(7): 934-942.

Hardin B O, Drnevich V P (1972) Shear modulus and damping in soils: design equations and curves. J Soil Mech Found Div, ASCE, 98(7): 667-692.

Heider Y, Avci O, Markert B, Ehlers W (2014) The dynamic response of fluid-saturated porous materials with application to seismically induced soil liquefaction. Soil Dyn Earthq Eng, 63: 120137.

Ishihara K, Towhata I (1983) Sand response to cyclic rotation of principal stress directions as induced by wave loads. Soils Found, 1983, 23(4): 11-26.

Ishihara K (1993) Liquefaction and flow failure during earthquakes. Géotechnique, 43(3): 351415.

Itasca Consulting Group Inc. 2002. FLAC3D, version 3.0. Itasca Consulting Group Inc., Minneapolis, Minn.

Jardine R J, Potts D M, Fourie A B, Burland J B (1986) Studies of the influence of nonlinear stress-strain characteristics in soil-structure interaction. Géotechnique, 36(3): 377-397.

Jeng D S (2001) Numerical modelling for wave-seabed-pipe interaction in a non-homogeneous porous seabed. Soil Dyn Earthq Eng, 21(8): 699-712.

Jeng D S. Porous Models for Wave-Seabed Interactions. Heidelberg: Springer, 2013.

Jeng D S, Seymour B R, Li J (2007) A new approximation for pore pressure accumulation in marine sediment due to water wave. Int J Numer Anal Methods Geomech, 31(1): 53-69.

Jeng D S, Zhao H Y (2015) Two-dimensional model for accumulation of pore pressure in marine sediments. J Waterw Port Coast Ocean Eng, 141(3): 04014042.

Kirca Ö, Sumer B M, Fredsøe J (2013) Seabed liquefaction under Standing waves. J Waterw Port Coast Ocean Eng, ASCE, 139(6): 489-501.

Kirca Ö, Sumer B M, Fredsøe J (2014) Influence of clay content on wave-induced liquefaction. J Waterw Port Coast Ocean Eng, ASCE, 140: 04014024, 1-11.

Luan M, Qu P, Jeng D S, Guo Y, Yang Q (2008) Dynamic response of a porous seabed-pipeline interaction under wave loading: Soil-pipeline contact effects and inertial effects. Comput Geotech, 
35: $173-186$.

Martin G R, Finn W D L, Seed H B (1975) Fundamentals of liquefaction under cyclic loading. J Geotech Engrg, ASCE, 101(GT5): 423-438.

Martin G R, Seed H B (1982) One-dimensional dynamic ground response analyses. J Geotech Engrg, ASCE, 108(7): 935-952.

Matasović N, Vucetic M (1993) Cyclic characterization of liquefiable sands. J Geotech Engrg, ASCE, 119(11): 1805-1822.

Miyamoto J, Sassa S, Sekiguchi H (2004) Progressive solidification of a liquefied sand layer during continued wave loading. Géotechnique, 54(10): 617-629.

Palmer A C (1996) A flaw in the conventional approach to stability design of pipelines. Proc, Offshore Pipeline Technology Conf, Amsterdam, The Netherlands.

Palmer A C, Teh T C, Bolton M D, Damgaard J S (2004) Stable pipelines on unstable seabed: Progress towards a rational design method. Proc, Offshore Pipeline Technology Conf, Amsterdam, The Netherlands.

Popescua R, Prevost J H, Deodatis G, Chakrabortty P (2006) Dynamics of nonlinear porous media with applications to soil liquefaction. Soil Dyn Earthq Eng, 26: 648-665.

Prèvost J H, Eide O, Andersen K H (1975) Wave induced pressures in permeable seabeds. J Waterw Harbors Coastal Eng, ASCE, 101(4): 464-465.

Pyke R M (1979) Nonlinear soil models for irregular cyclic loadings. J Geotech Engrg, ASCE, 105(GT6): 715-726.

Sassa S, Sekiguchi H (1999) Wave-induced liquefaction of beds of sand in a centrifuge. Géotechnique, 49(5): 621-38.

Sassa S, Sekiguchi H (2001) Analysis of wave-induced liquefaction of sand beds. Géotechnique, 51(2): 115-126.

Sawicki A, Mierczyński J (2006) Developments in modelling liquefaction of granular soils, caused by cyclic loads. Appl Mech Rev, 59 (2): 91-106.

Seed H B, Rahman M S (1978) Wave-induced pore pressure in relation to ocean floor stability of cohesionless soils. Marine Geotechnol, 3(2): 123-50.

Small J C, Booker J R, Davis E H (1976) Elasto-plastic consolidation of soils. Int J Solids Struct, 12: 431-448.

Stewart J P, On-Lei Kwok A, Hashash Y M A, Matasović N, Pyke R, Wang Z L, Yang Z H (2008) Benchmarking of nonlinear geotechnical ground response analysis procedures. Peer Report, University of California, Berkeley.

Sumer B M. Liquefaction around marine structures: (With CD-ROM). World scientific, 2014.

Sumer B M, Fredsøe J. The mechanism of scour in the marine environment. New Jersey: World Scientific, 2002.

Sumer B M, Hatipoglu F, Fredsøe J, Hansen N E O (2006a) Critical flotation density of pipelines in soils liquefied by waves and density of liquefied soils. J Waterway Port Coastal Ocean Eng, 132(4): 252-265.

Sumer B M, Hatipoglu F, Fredsøe J, Sumer S K (2006b) The sequence of sediment behaviour during wave-induced liquefaction. Sedimentology, 53(3): 611-629.

Sumer B M, Kirca Ö, Fredsøe J (2012) Experimental validation of a mathematical model for seabed liquefaction under waves. Int J Offshore Polar Eng, 22(2), 133-141.

Sumer B M, Truelsen C, Fredsøe J (2006c) Liquefaction around pipelines under waves. J Waterw 
Teh T C, Palmer A C, Bolton M D, Damgaard J (2006) Stability of submarine pipelines on liquefied seabeds. J Waterw Port Coast Ocean Eng, ASCE, 132(4): 244-251.

Teh T C, Palmer A C, Damgaard J S (2003) Experimental study of marine pipelines on unstable and liquefied seabed. Coast Eng, 50: 1-17.

Tian Y H, Cassidy M J, Gaudin C (2010) Advancing pipe-soil interaction models in calcareous sand. Appl Ocean Res, 32: 284-297.

Ulker M B C, Rahman M S (2009). Response of saturated porous media: Different formulations and their validity. Int J Numer Anal Methods Geomech, 33(5): 633-664.

Vucetic M, Dobry R (1991) Effect of soil plasticity on cyclic response. J Geotech Eng, ASCE, 117(89): 89-107.

Zhang X L, Xu C S, Han Y (2015) Three-dimensional poro-elasto-plastic model for wave-induced seabed response around submarine pipeline. Soil Dyn Earthq Eng, 69: 163-171.

Zhao H Y, Jeng D S, Guo Z, Zhang J S (2014) Two dimensional model for pore pressure accumulations in the vicinity of a buried pipeline. J Offshore Mech Arct Eng, ASME, 136(4): 042001 .

Zhao H Y, Jeng D S (2016) Accumulated Pore Pressures around Submarine Pipeline Buried in Trench Layer with Partial Backfills. J Eng Mech, 04016042.

Zhao H Y, Jeng D S, Liao C C (2016) Effects of cross-anisotropic soil behaviour on the waveinduced residual liquefaction in the vicinity of pipeline buried in elasto-plastic seabed foundations. Soil Dyn Earthq Eng, 80: 40-55.

Zienkiewicz O C, Chang C T, Hinton E (1978) Non-Linear seismic response and liquefaction, Int J Numer Analyt Meth Geomech, 2: 381-404.

Zienkiewicz O C, Chang C T, Bettess P (1980) Drained, undrained, consolidating and dynamic behavior assumptions in soils. Géotechnique, 30 (4): 385-395. 


\section{Figure captions}

Figure 1: Sketch of wave-seabed-pipeline interaction.

Figure 2: Mechanisms of wave-induced excess pore pressures (not in scale).

Figure 3: Schematic illustration of stress-strain behavior in first cycle (starting at time $t=0$ ) and subsequent cycle (at time $t$ ).

Figure 4: Grid layout of computation domain in detail.

Figure 5: Schematic illustration of loading scheme for single element test.

Figure 6: Measured and calculated cyclic stress-strain loops of the natural Nanjing fine sand in cyclic loading test.

Figure 7: Comparison of wave-induced pore pressure response versus time between the present model and the wave flume test by Sumer et al. (2006c): a) at the bottom of the pipe; and b) in the far field at the same level.

Figure 8: Shear stiffness and damping ratio decay curves of marine silt with $D_{\mathrm{r}}=50 \%$.

Figure 9: Time histories of wave-induced pore pressure along the perimeter of the pipeline under wave loadings with and without the nonlinear component: a) at the top; b) at the side edge; and c) at the bottom of the pipeline.

Figure 10: Time histories of development of liquefaction zone in the vicinity of the pipeline under a linear wave (on the left side) and under a nonlinear wave (on the right side).

Figure 11: Time histories of wave-induced pore pressure along the perimeter of the pipeline and in the far field at the corresponding levels under nonlinear wave loading: a) at the top; b) at the side edge; and c) at the bottom of the pipeline.

Figure 12: Predicted effective stress path: a) at the bottom of the pipeline; and b) in the far field at the same level.

Figure 13: Distribution of initial vertical effective stress in the consolidation status.

Figure 14: Predicted deviatoric stress path: a) at the bottom of the pipeline; and b) in the far field at the same level.

Figure 15: Predicted shear stress-strain response: a) at the bottom of the pipeline; and b) in the far field at the same level. 
Figure 1

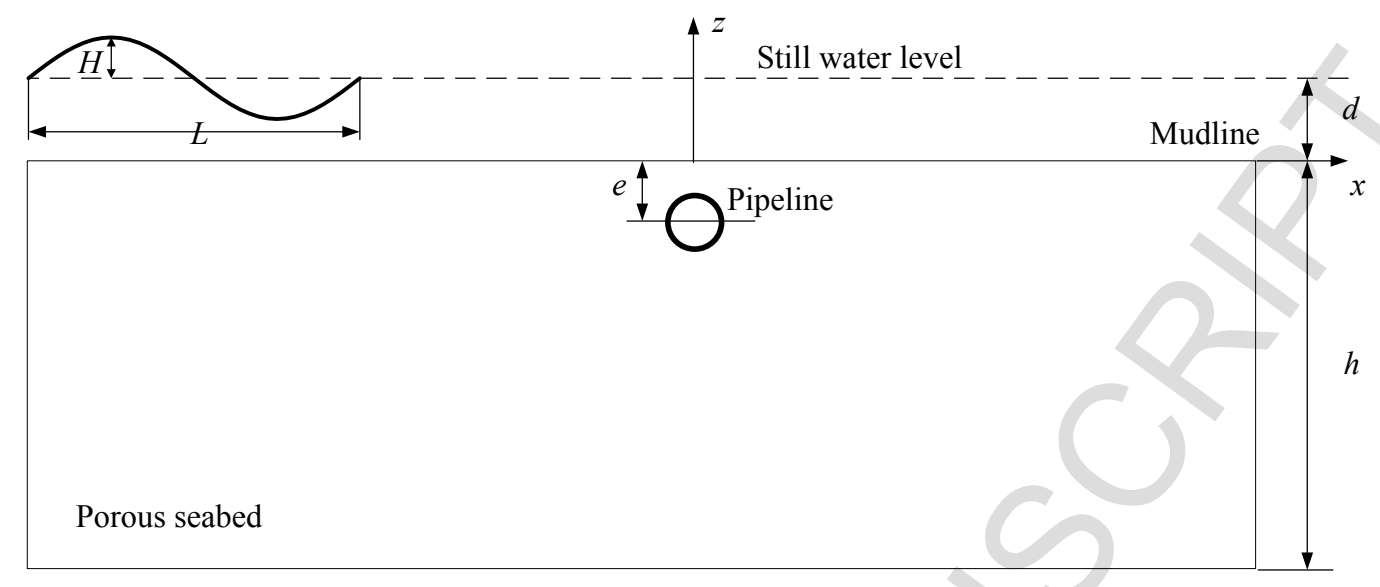

Rigid impermeable bottom 
Figure 2

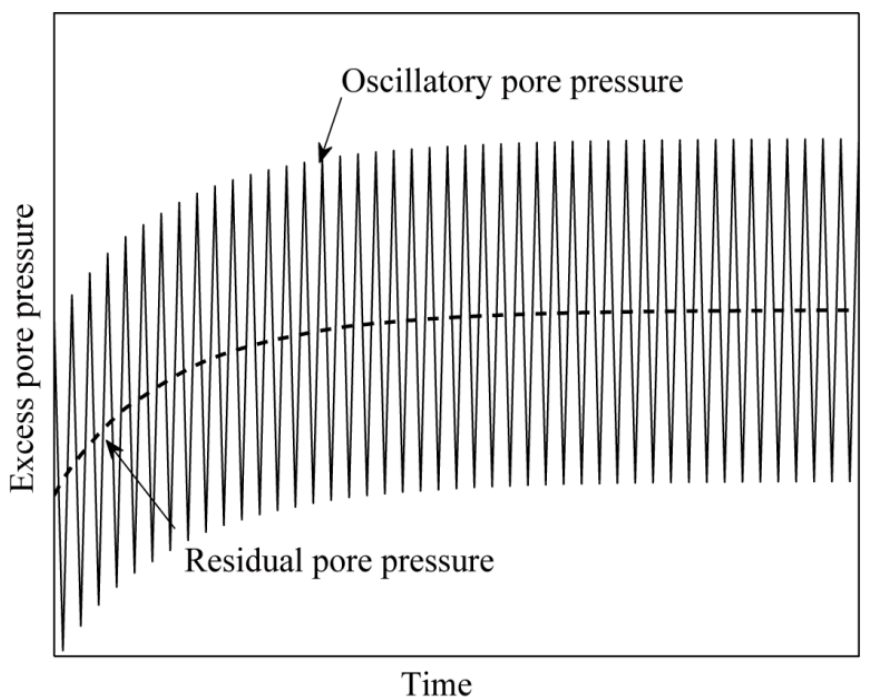

Time 
Figure 3

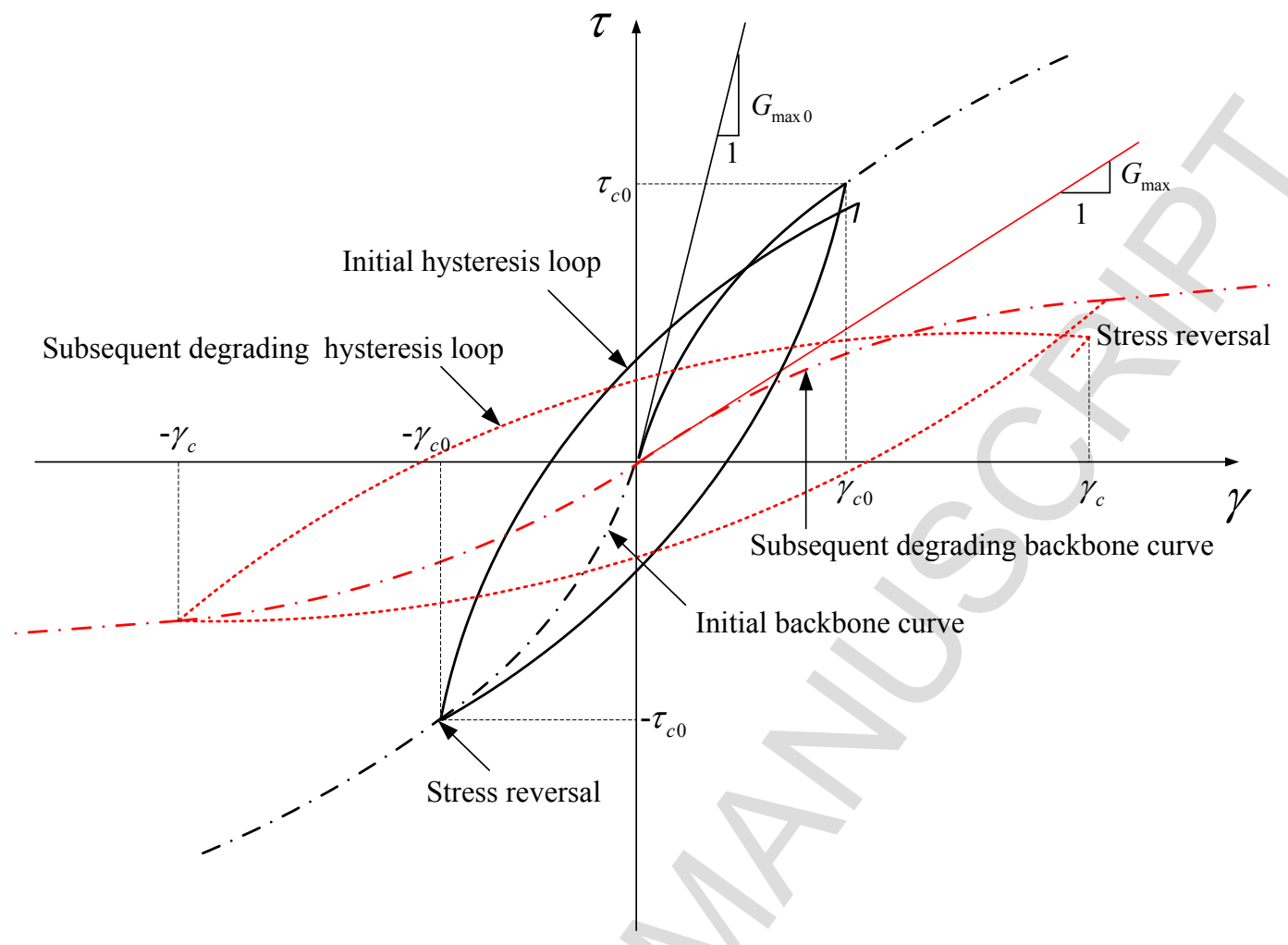


Figure 4

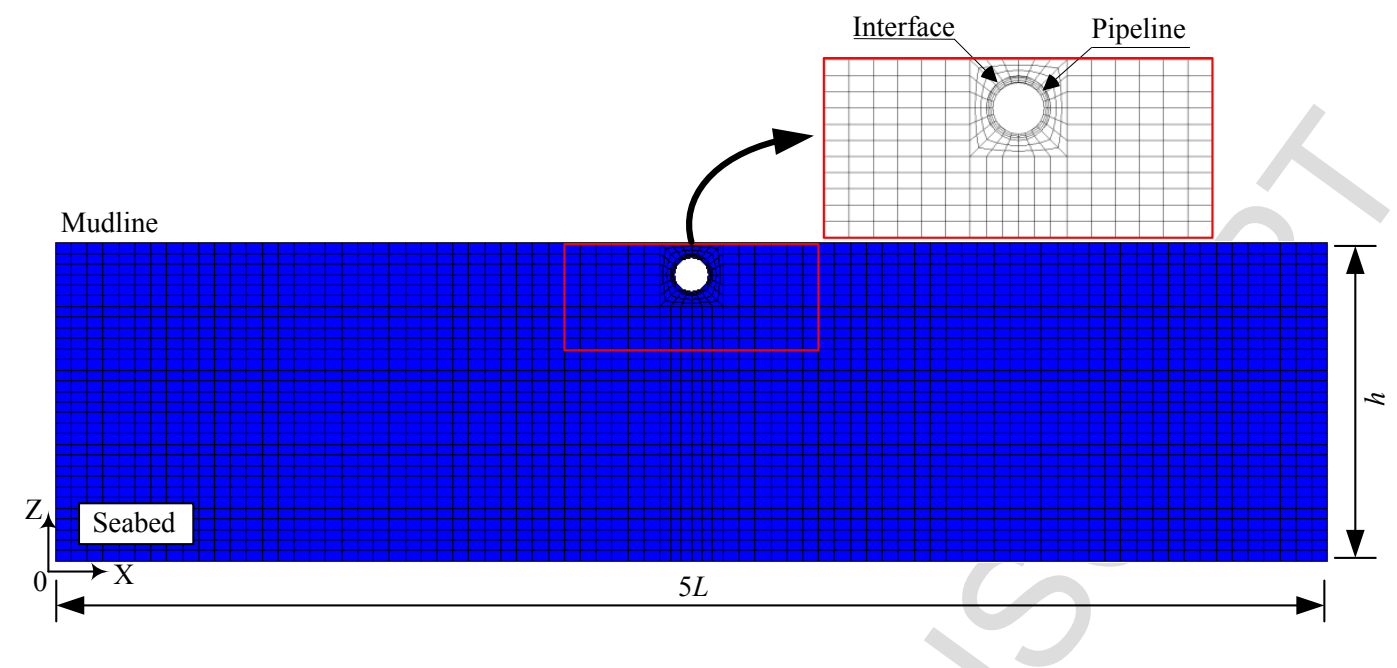


Figure 5<smiles></smiles>

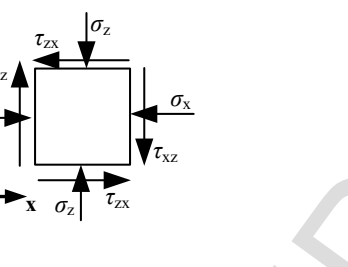


Figure 6
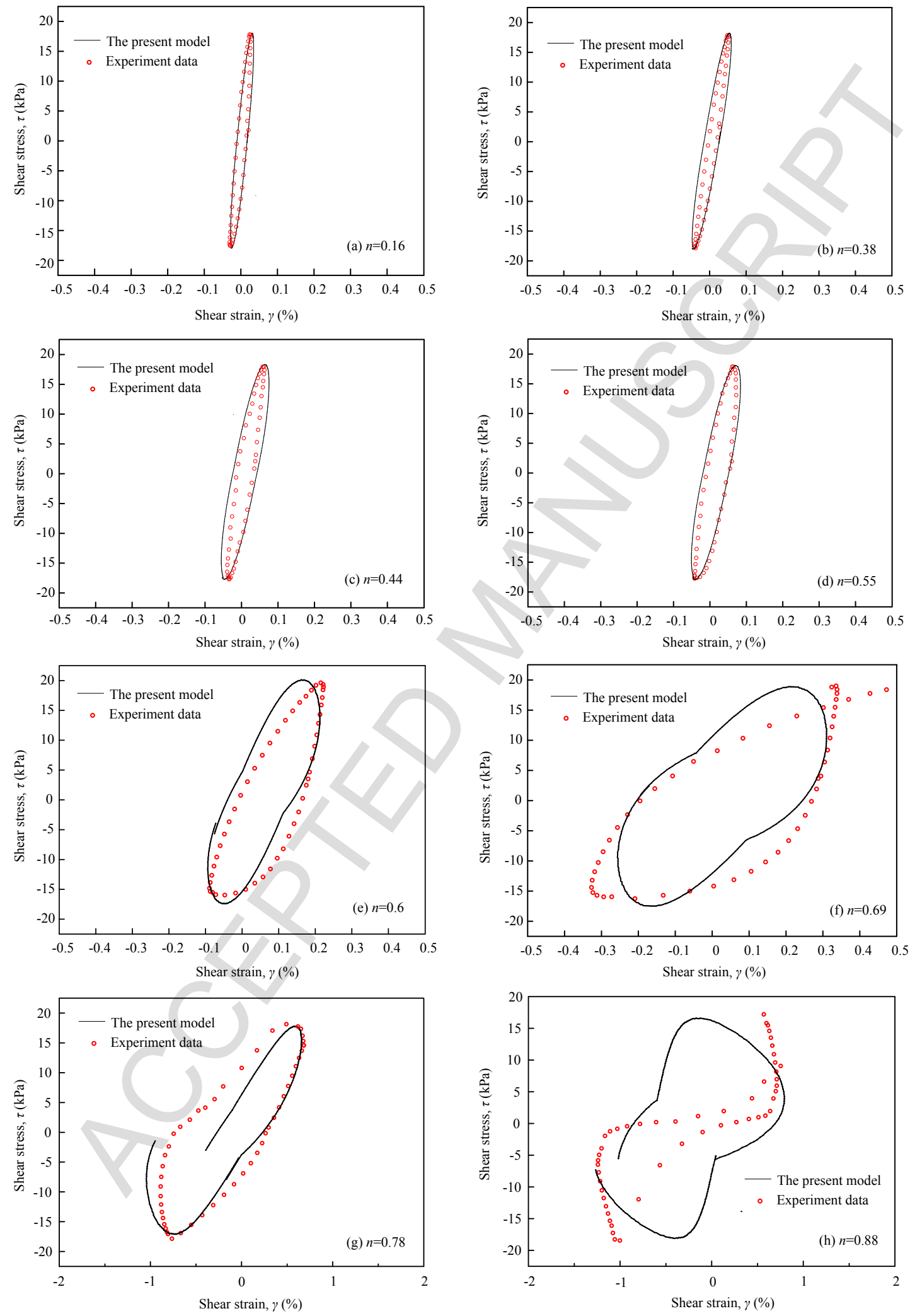
Figure 7
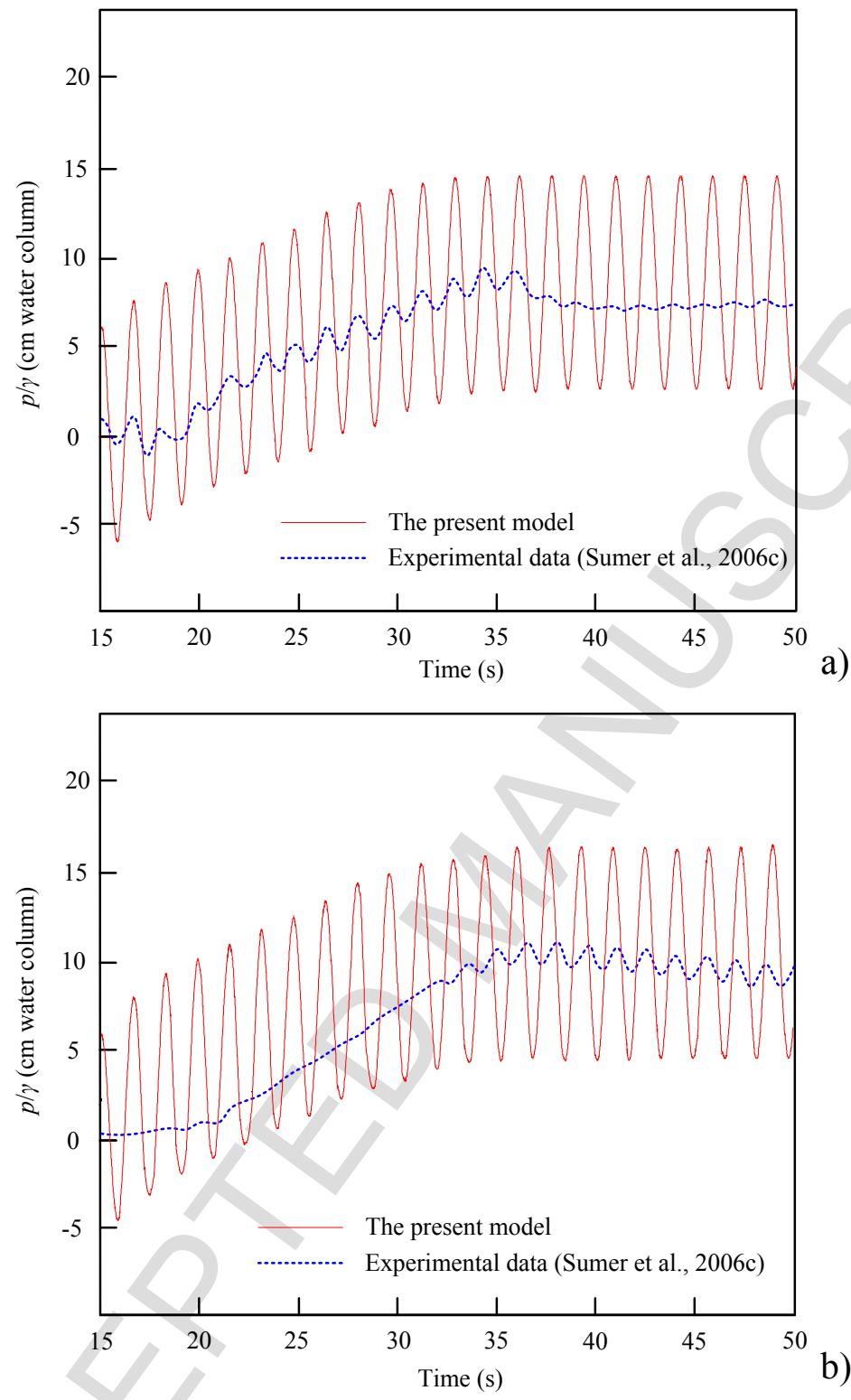
Figure 8

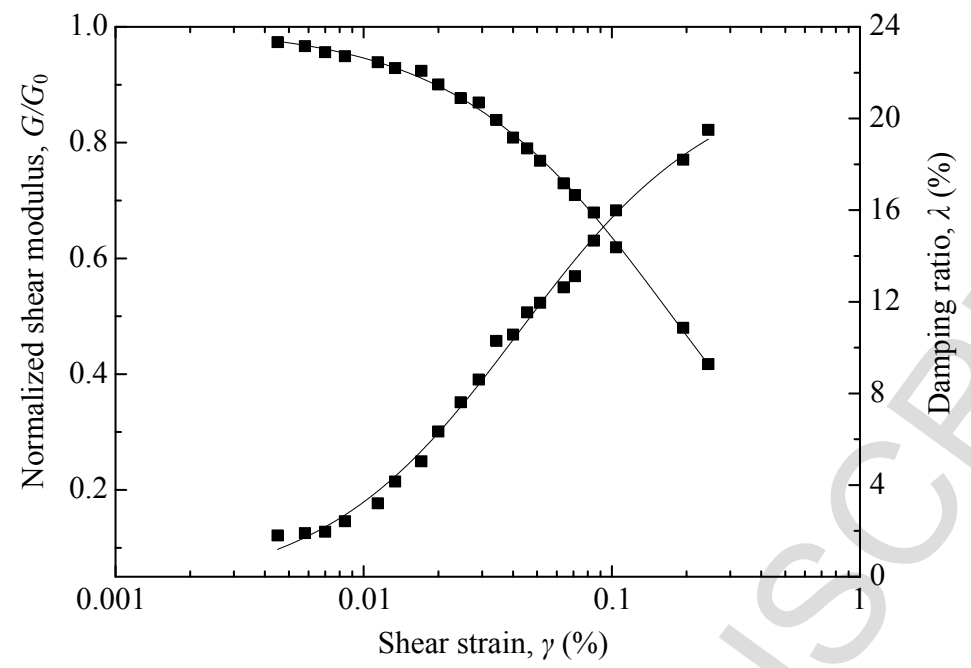


Figure 9
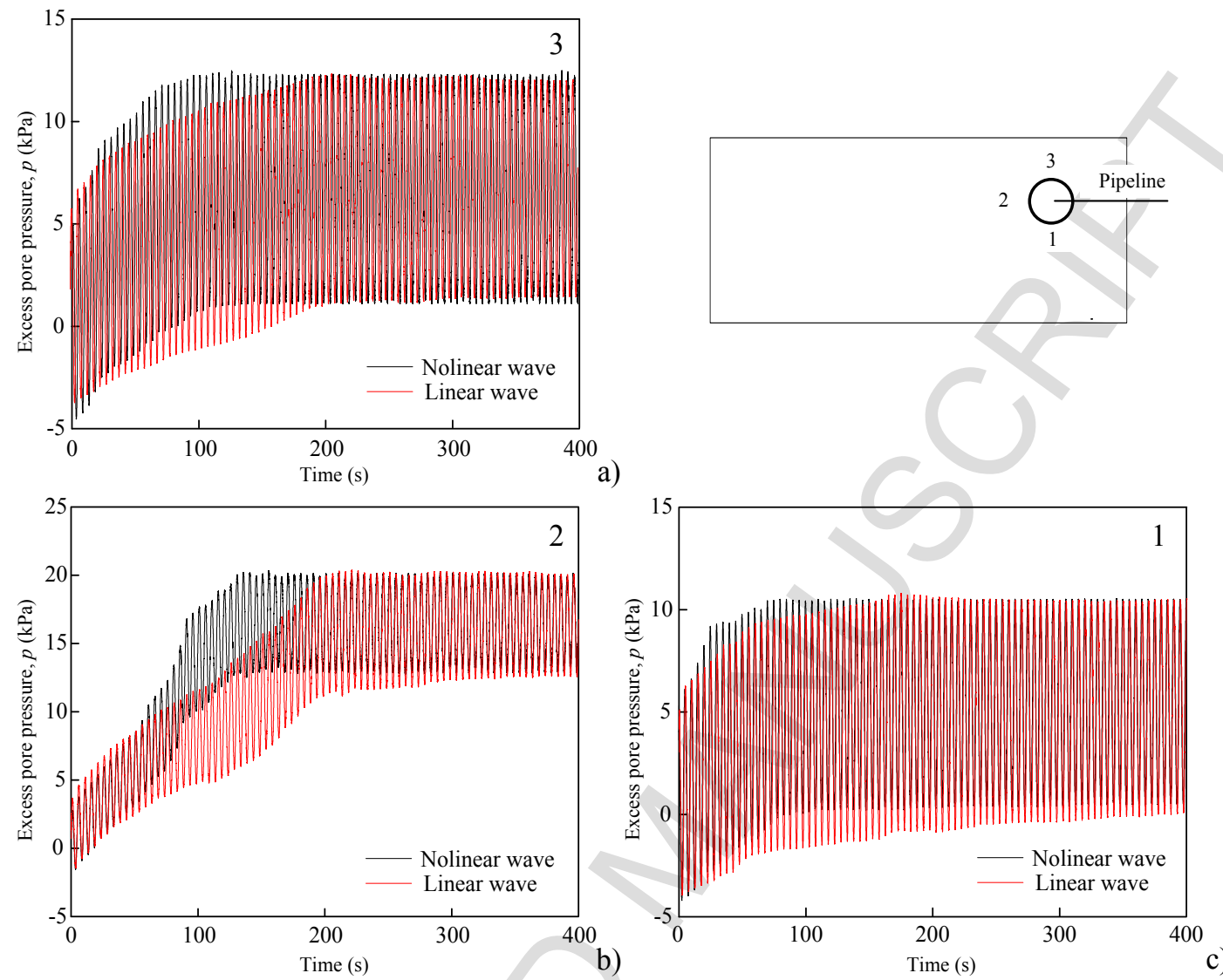
Figure 10

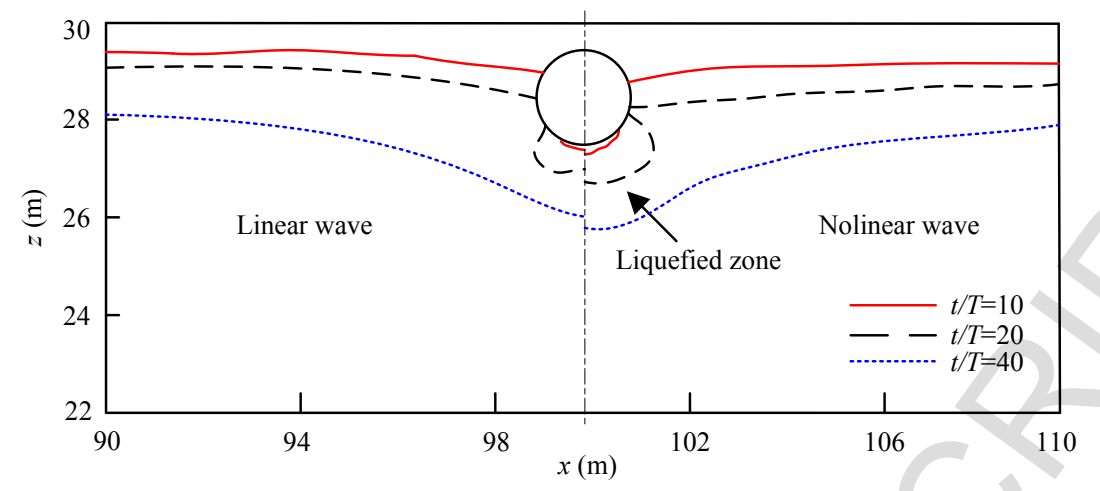


Figure 11
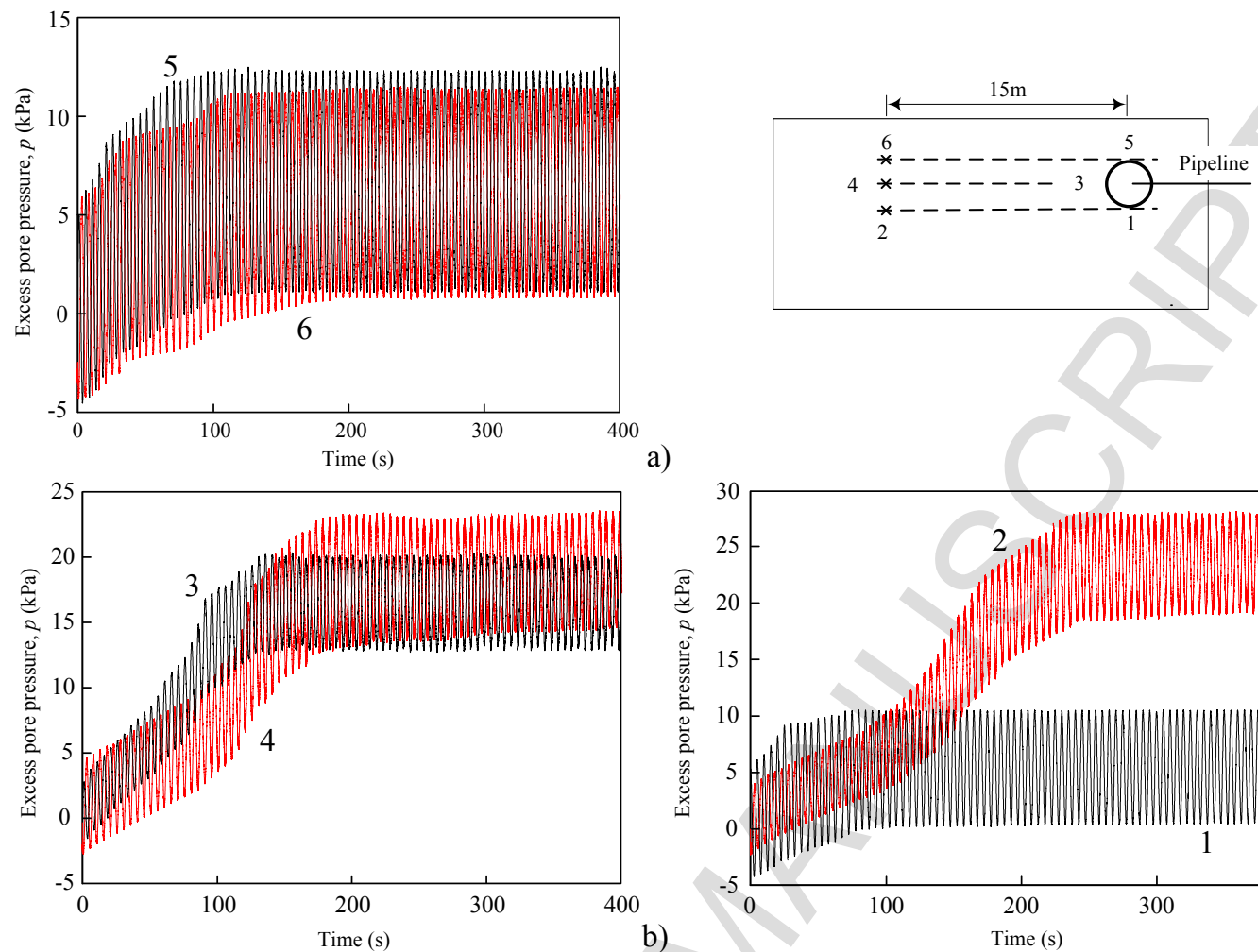

a)

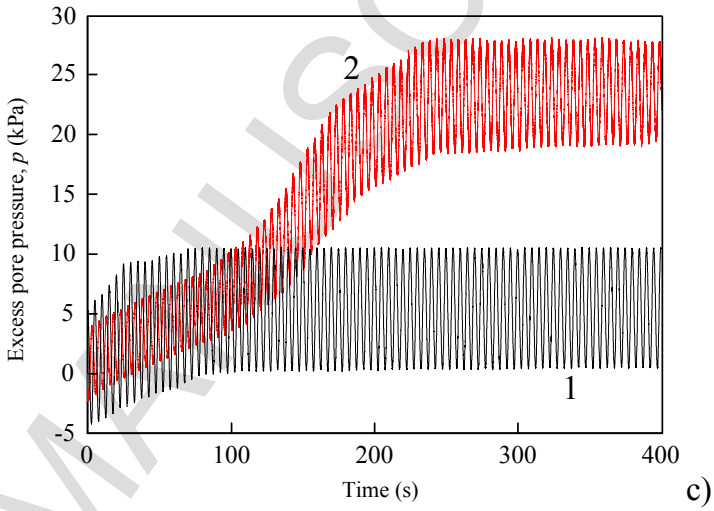


Figure 12
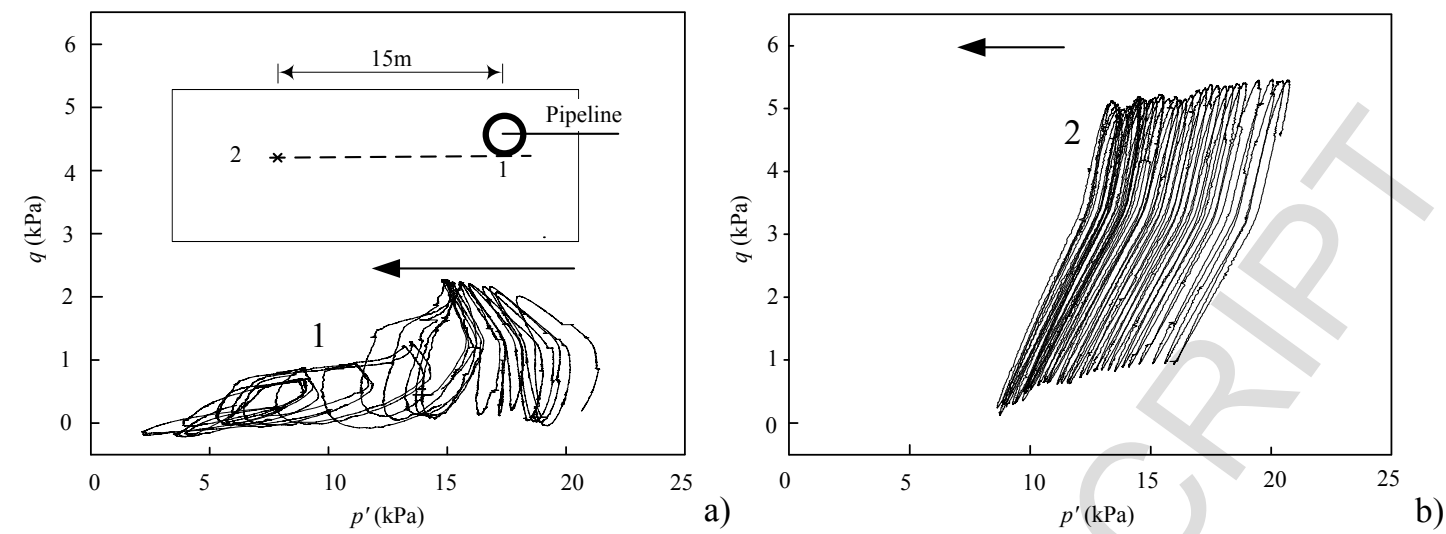
Figure 13

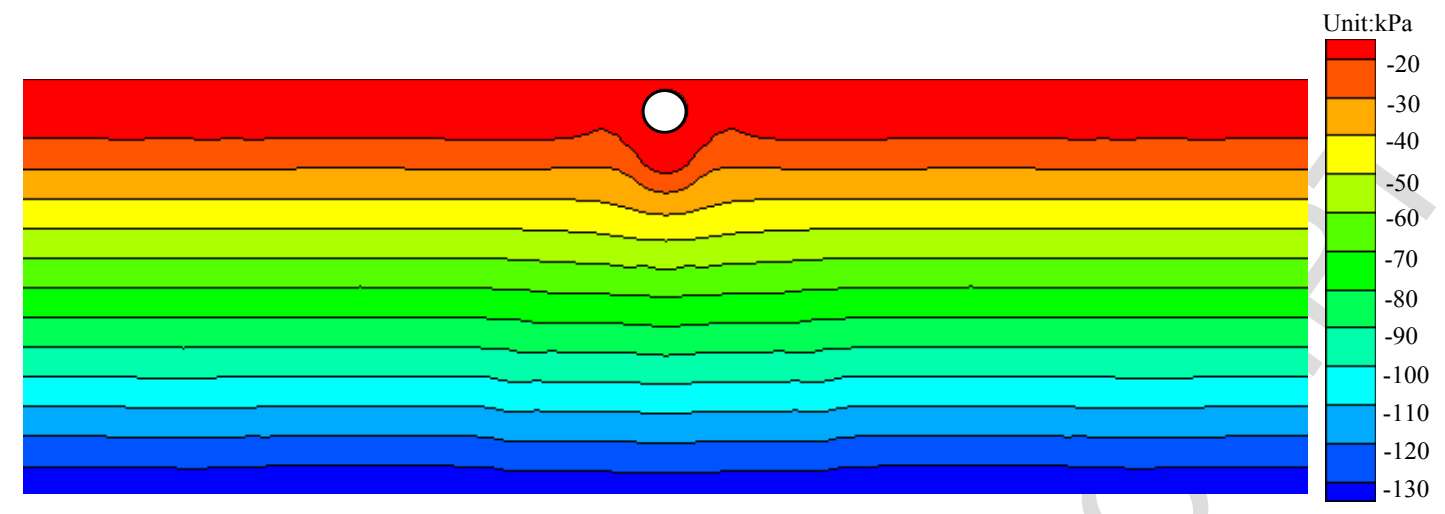


Figure 14
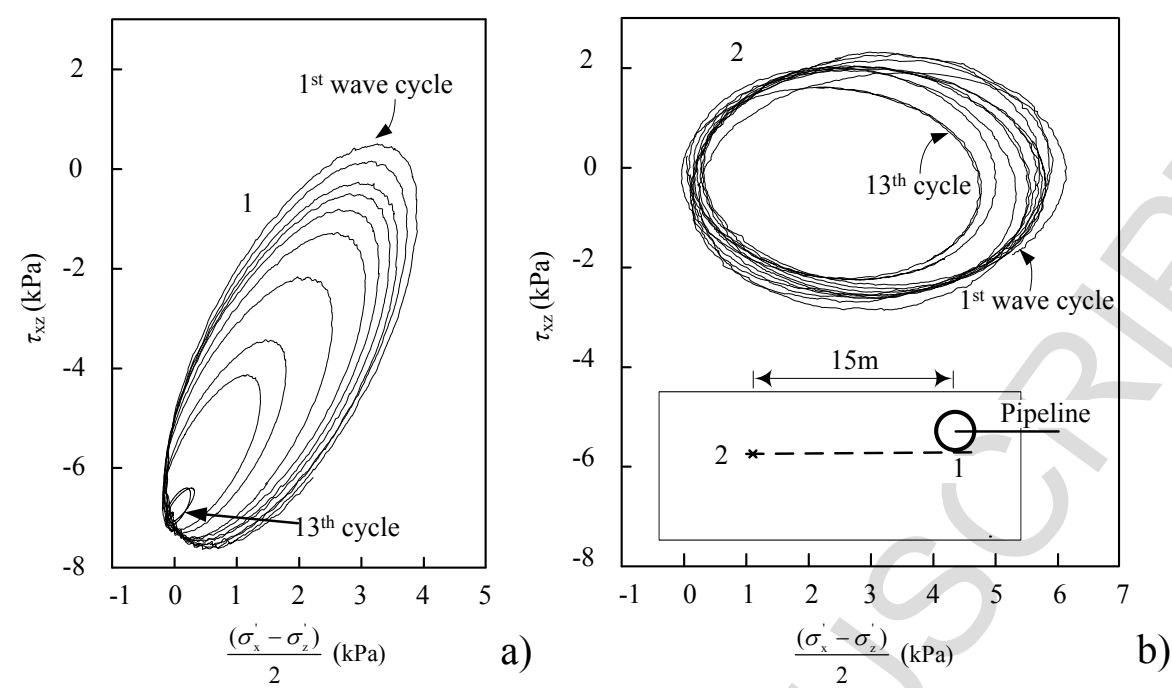
Figure 15
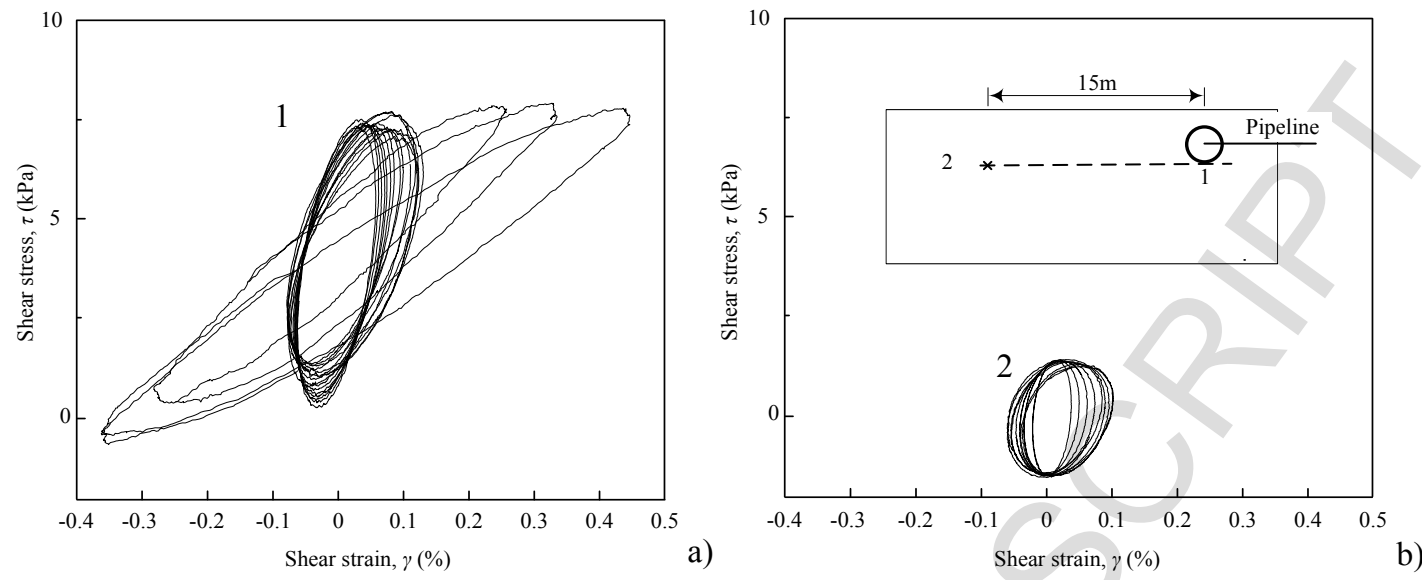


\section{Table captions}

Table 1: Soil parameters used in the model calibration for individual element behavior

Table 2: Input data for model calibration against the wave flume test by Sumer et al. (2006c)

Table3: Input data for numerical examples 


\section{Table 1}

\begin{tabular}{|c|c|}
\hline Characteristics & Value \\
\hline \multicolumn{2}{|c|}{ Seabed } \\
\hline Soil porosity $\left(n_{\mathrm{s}}\right)$ & 0.50 \\
\hline Poisson's ratio $(v)$ & 0.30 \\
\hline Reference initial shear modulus $\left(G_{\max 0}^{r e f}\right)$ & $5 \times 10^{7}\left(\mathrm{~N} / \mathrm{m}^{2}\right)$ \\
\hline Submerged specific weight of soil $\left(\gamma^{\prime}\right)$ & $9.2\left(\mathrm{kN} / \mathrm{m}^{3}\right)$ \\
\hline Degree of saturation $\left(S_{\mathrm{r}}\right)$ & 1 \\
\hline Relative density $\left(D_{\mathrm{r}}\right)$ & $50 \%$ \\
\hline \multicolumn{2}{|c|}{ Davidenkov model parameters } \\
\hline A & 1.02 \\
\hline B & 0.40 \\
\hline$\gamma_{0} /\left(\times 10^{-4}\right)$ & 4 \\
\hline$C_{1}$ & 0.43 \\
\hline$C_{2}$ & 0.93 \\
\hline
\end{tabular}




\section{Table 2}

\begin{tabular}{|c|c|}
\hline Characteristics & Value \\
\hline \multicolumn{2}{|c|}{ Seabed } \\
\hline Soil porosity $\left(n_{\mathrm{s}}\right)$ & 0.35 \\
\hline Poisson's ratio $(v)$ & 0.35 \\
\hline initial tangent shear modulus $\left(G_{\max 0}\right)$ & $5.4 \times 10^{6}\left(\mathrm{~N} / \mathrm{m}^{2}\right)$ \\
\hline Soil permeability $(k)$ & $10^{-5}(\mathrm{~m} / \mathrm{s})$ \\
\hline Coefficient of lateral earth pressure $\left(K_{0}\right)$ & 0.41 \\
\hline Submerged specific weight of soil $\left(\gamma^{\prime}\right)$ & $10.73\left(\mathrm{kN} / \mathrm{m}^{3}\right)$ \\
\hline Degree of saturation $\left(S_{\mathrm{r}}\right)$ & 1 \\
\hline Grain size $\left(d_{50}\right)$ & 0.045 \\
\hline Thickness $(h)$ & $0.17(\mathrm{~m})$ \\
\hline \multicolumn{2}{|c|}{ Davidenkov model parameters } \\
\hline$A$ & 1.02 \\
\hline$B$ & 0.40 \\
\hline$\gamma_{0} /\left(\times 10^{-4}\right)$ & 3.8 \\
\hline$C_{1}$ & 0.114 \\
\hline$C_{2}$ & 3.5 \\
\hline \multicolumn{2}{|c|}{ Wave } \\
\hline Wave Height $(H)$ & $16.6(\mathrm{~cm})$ \\
\hline Wave period $(T)$ & $2.30(\mathrm{~s})$ \\
\hline Water depth $(d)$ & $42(\mathrm{~cm})$ \\
\hline \multicolumn{2}{|c|}{ Pipeline characteristics } \\
\hline Outer diameter $(D)$ & $8(\mathrm{~cm})$ \\
\hline Pipe position $(e)$ & $5(\mathrm{~cm})$ \\
\hline Young's modulus $(E)$ & $6.9 \times 10^{10}\left(\mathrm{~N} / \mathrm{m}^{2}\right)$ \\
\hline Poisson's ratio $(v)$ & 0.18 \\
\hline Density $(\rho)$ & $2700\left(\mathrm{~kg} / \mathrm{m}^{3}\right)$ \\
\hline Buried depth $(h)$ & $6.50(\mathrm{~cm})$ \\
\hline
\end{tabular}




\section{Table 3}

\begin{tabular}{|c|c|}
\hline Characteristics & Value \\
\hline \multicolumn{2}{|c|}{ Seabed } \\
\hline Soil porosity $\left(n_{\mathrm{s}}\right)$ & 0.50 \\
\hline Poisson's ratio $(v)$ & 0.30 \\
\hline initial tangent shear modulus $\left(G_{\max 0}\right)$ & $6.5 \times 10^{7}\left(\mathrm{~N} / \mathrm{m}^{2}\right)$ \\
\hline Soil permeability $(k)$ & $10^{-5}(\mathrm{~m} / \mathrm{s})$ \\
\hline Coefficient of lateral earth pressure $\left(K_{0}\right)$ & 0.42 \\
\hline Submerged specific weight of soil $\left(\gamma^{\prime}\right)$ & $\left.\mathrm{N} / \mathrm{m}^{3}\right)$ \\
\hline Degree of saturation $\left(S_{\mathrm{r}}\right)$ & 1 \\
\hline Relative density $\left(D_{\mathrm{r}}\right)$ & $50 \%$ \\
\hline Thickness $(h)$ & $30(\mathrm{~m})$ \\
\hline \multicolumn{2}{|c|}{ Davidenkov model parameters } \\
\hline A & 1.03 \\
\hline B & 0.50 \\
\hline$\gamma_{0} /\left(\times 10^{-4}\right)$ & 1.81 \\
\hline$C_{1}$ & 0.43 \\
\hline$C_{2}$ & 0.93 \\
\hline \multicolumn{2}{|c|}{ Wave } \\
\hline Wave Height $(H)$ & $2(\mathrm{~m})$ \\
\hline Wave period $(T)$ & $5(s)$ \\
\hline Water depth $(d)$ & $5(\mathrm{~m})$ \\
\hline \multicolumn{2}{|c|}{ Pipeline characteristics } \\
\hline Outer diameter $(D)$ & $2(\mathrm{~m})$ \\
\hline Young's modulus $(E)$ & $2.06 \times 10^{10}\left(\mathrm{~N} / \mathrm{m}^{2}\right)$ \\
\hline Poisson's ratio $(v)$ & 0.18 \\
\hline Density $(\rho)$ & $2200\left(\mathrm{~kg} / \mathrm{m}^{3}\right)$ \\
\hline Buried depth $(h)$ & $1.50(\mathrm{~m})$ \\
\hline
\end{tabular}




\section{Highlights:}

- A cyclic characterization method is proposed for wave-liquefiable seabed-pipeline interactions.

- Shear-volume coupling behavior is incorporated into a well-calibrated cyclic stress-strain model.

- Model calibrations are carried out in terms of soil element behavior and seabed response.

- Typical results on the wave-induced dynamics of pipeline in liquefiable seabed are interpreted. 


\section{Accepted Manuscript}

Wave-induced dynamics of marine pipelines in liquefiable seabed

Kai Zhao, Hao Xiong, Guoxing Chen, Dingfeng Zhao, Weiyun Chen, Xiuli Du

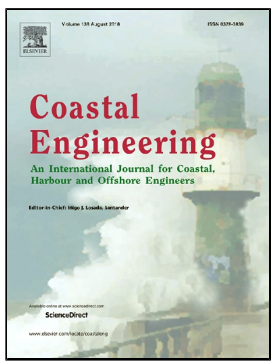

PII:

S0378-3839(17)30244-2

DOI:

10.1016/j.coastaleng.2018.06.007

Reference:

CENG 3393

To appear in:

Coastal Engineering

Received Date:

05 May 2017

Accepted Date:

24 June 2018

Please cite this article as: Kai Zhao, Hao Xiong, Guoxing Chen, Dingfeng Zhao, Weiyun Chen, Xiuli Du, Wave-induced dynamics of marine pipelines in liquefiable seabed, Coastal Engineering (2018), doi: 10.1016/j.coastaleng.2018.06.007

This is a PDF file of an unedited manuscript that has been accepted for publication. As a service to our customers we are providing this early version of the manuscript. The manuscript will undergo copyediting, typesetting, and review of the resulting proof before it is published in its final form. Please note that during the production process errors may be discovered which could affect the content, and all legal disclaimers that apply to the journal pertain. 